\title{
Cylindrical Magnetic Nanowires Applications
}

\author{
Julián A. Moreno ${ }^{1}$, Cristina Bran², Manuel Vazquez², Jürgen Kosel ${ }^{3}$ \\ ${ }^{1}$ Physical Science and Engineering Division \\ King Abdullah University of Science and Technology (KAUST), Thuwal 23955-6900, Saudi Arabia \\ ${ }^{2}$ Instituto de Ciencia de Materiales de Madrid, CSIC, Madrid, 28049, Spain \\ ${ }^{3}$ Computer Electrical and Mathematical Science and Engineering Division \\ King Abdullah University of Science and Technology (KAUST), Thuwal 23955-6900, Saudi Arabia
}

\begin{abstract}
Cylindrical magnetic nanowires feature unique properties, which make them attractive for fundamental research as well as novel applications. These one-dimensional structures introduce a pronounced shape anisotropy that together with material selection can strongly affect the magnetic properties and can be tuned by incorporating segments of different materials or diameters along the length. They attract a large interest in the scientific community, ranging from physicists to material scientists to bioengineers. Consequently, these nanowires are developed for and employed in very diverse applications in medicine, biology, data and energy storage, catalysis or microwave electronics, among others. In this review we investigate the most active emerging applications of cylindrical nanowires grown in alumina templates by electrochemical deposition. This method has several key features, including low cost and a high level of control over the design. A fundamental property that distinguishes those applications is the operating frequency, which we chose to apply as an underlying structure for this review. With this we attempt to provide a wide and organized view of applications based on cylindrical magnetic nanowires with a focus on tailored physical and chemical properties.
\end{abstract}

Index Terms - cylindrical magnetic nanowires, nanotechnology, electroplating, sensors, devices, biomedical, passive electronic elements.

\section{INTRODUCTION}

$\mathrm{C}$ YLINDRICAL MAGNETIC nanowires (NWs) are one dimensional structures with unique fundamental properties and a wide range of novel applications. A high aspect ratio leads to large shape anisotropies, which can dominate the magnetic properties and magnetization processes. Their geometry causes a curvature-induced effective anisotropy or chiral symmetry breaking, and favors the formation of uncommon magnetic textures, contrary to more common planar nano-strips [1]-[4]. Several reviews have been published, mostly in the form of book chapters focusing on fabrication and characterization of NWs, yet there are relatively few documents with concise emphasis on the working of devices (regardless of their technological readiness). For instance, the latest works from Mohammed et al [5], Xu et al [6], Staňo \& Fruchart [7], L. Piraux [8] and the book edited by Vazquez [9], amongst others, are excellent references to understand the state-of-the-art of fabrication, characterization and magnetization control of NWs, setting the background of fundamental aspects. The main purpose of this review is to compile and describe the most recent applications based on NWs. Although there are several techniques to grow NWs such as using few nanometer-diameter nuclear-track etched polymer templates [8], growing them individually using Focused Electron Beam Induced Deposition (FEBID) [10] and directly released in solution using soft, polyol or organometallic chemistry [11], to name a few, we will limit our considerations to those works, in which the NWs were fabricated by electrochemical deposition into alumina templates. This last-mentioned method provides a large control over parameters in combination with many degrees of freedom for tailored designs.
These applications exploit either internal magnetization changes or external torques on individual or collective NWs with single or multi-domain states at certain frequencies:

- They can act as a static source of magnetic field [12] and therefore be detected by e.g. magnetoresistive sensors [13], [14], serve as highly sensitive magnetic force microscopy (MFM) tips [15] or induce field fluctuations in energy harvesters [16], [17].

- Hybrid magnetic / piezoelectric, magnetoelectric and magnetic nanowires are proposed for bioengineering applications as nano-robots and nano-swimmers for biomedical applications [18], [19], or for security code sensors [20].

- They can be excited with lasers or alternating magnetic fields up to hundreds of kilohertz and produce motion and/or heat [21]-[23]. This makes them attractive for biomedical applications [24], targeting cancer cells when they are properly functionalized [25], [26] or as MRI contrast agents working at around hundredths of megahertz [27], [28].

- Arrays of NWs have been used as electromagnetic pulse detectors [3], microwaves circulators or phase shifters [29]-[32] at $10-40 \mathrm{GHz}$ frequencies.

- When isolated, effects like giant magneto-resistance (GMR) [33]-[35], domain wall (DW) pinning [36][38] and spin transfer torque (STT) [39]-[41] are exploited mainly for prospective memory applications [42]-[45] employing nanoseconds (GHz) pulses.

Fig 1 illustrates some of these applications at their range of operation frequency. Notice there is a gap from around 


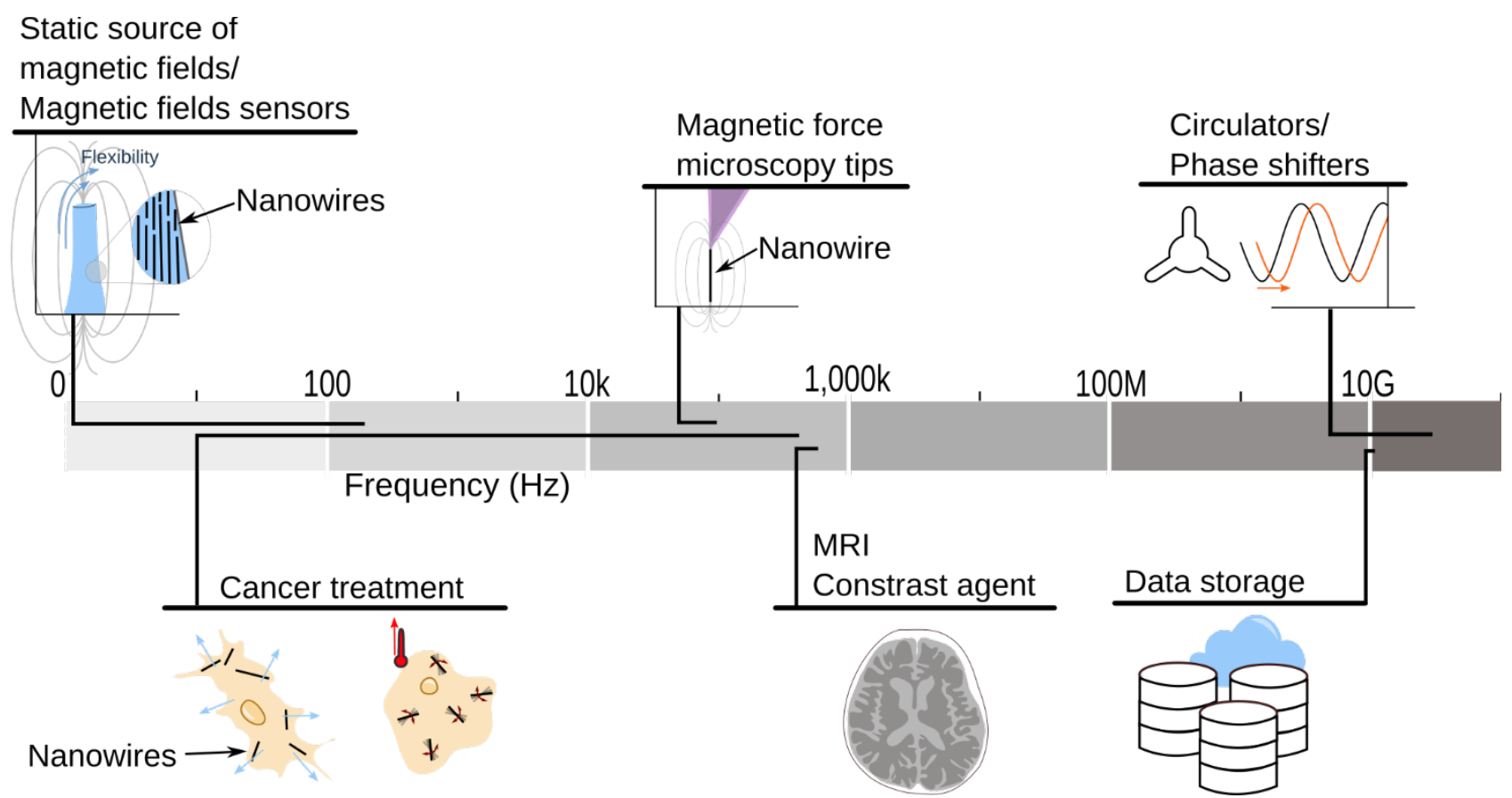

Fig 1. Overview of the wide range of applications of cylindrical magnetic nanowires and the operation frequencies.

$1000 \mathrm{kHz}(1 \mathrm{MHz})$ to the $\mathrm{GHz}$ regime. These are frequencies too big to induce significant mechanical movement or to be approved for use in living organisms and too small for electronics devices. Classifying applications in terms of the used frequency, either applied (external excitation) or internal (magnetization changes), as in $\mathbf{F i g} \mathbf{1}$, aims to provide a structured view of the field from the fundamental research to the design/engineering perspective.

\section{FABRICATION OF CYLINDRICAL NANOWIRES}

One of the most common and reproducible ways of fabricating NWs is by electrochemical deposition into anodized alumina templates. These templates consist of cylindrical nanochannels through an alumina bulk with a high regularity in length and diameter. Anodization of aluminum provides a wide range of channel diameters and inter-channel distances, and some control over the geometry of the channels like diameter modulations along the length [46]-[48]. Electrodeposition of metals into these templates results in the metal filling the channels and replicating their shape. The resulting NWs can be studied inside the template or they can be partly or entirely released from it by etching the alumina. These templates also contain a high density of channels (in the order of $10^{10}$ channels $/ \mathrm{cm}^{2}$ ) favoring high density arrays and fabrication yield. Therefore, these templates have been adopted for numerous applications and are widely used by research groups.

Most of the tailored properties of individual and collective NWs are inherited from the shape of the template and electrodeposition conditions. Different energy contributions define the magnetization behavior in a magnetic material, namely shape and crystal anisotropies, exchange interaction, the energy related to a self-demagnetizing field and external fields like magnetostatic interactions among neighboring nanowires. The shape of the template and length of the nanowires defines their shape anisotropy and overall demagnetizing field, while the nature of the electrodeposited metal and the electroplating conditions define the magnetocrystalline anisotropy. This latter energy term, for example, can be tuned by a suitable choice of the $\mathrm{pH}$ value of the electrolyte [49] or the deposition voltage and temperature [50].

\section{A. Fabrication of Anodized Alumina templates}

The most common way of fabricating alumina templates is by anodization - controlled oxidation of pure aluminum in one of three main electrolytes: sulfuric, oxalic or phosphoric acid driven by an electric field applied between a platinum mesh (counter electrode) and aluminum itself (working electrode) [51]. Oxidation conditions, such as temperature, electrolyte concentration and $\mathrm{pH}$ determine the rate at which the oxide layer is formed at the aluminum/oxide interface and dissolved into the electrolyte at the oxide/electrolyte interface. In the first anodization step (Fig. 2a) channels appear at random locations throughout the surface and progress towards the aluminum. These first channels appear mainly due to non-uniform oxide growth caused by the barrier oxide layer growing under tensile stress, creating cracks, where the electric field concentrates (in addition to local imperfections) facilitating oxide dissolution [48]. As this process continues, the channels increase their diameter approaching each other and depleting the aluminum sideways, eventually leading to a vertical growth in a hexagonal pattern. This depletion of the aluminum and the steady state applied electric field, define the inter-channel distance and channel diameter (Fig. 2a - c). These channels are also referred to as pores, even when they do not run all the way through the alumina, i.e., with both ends open. 


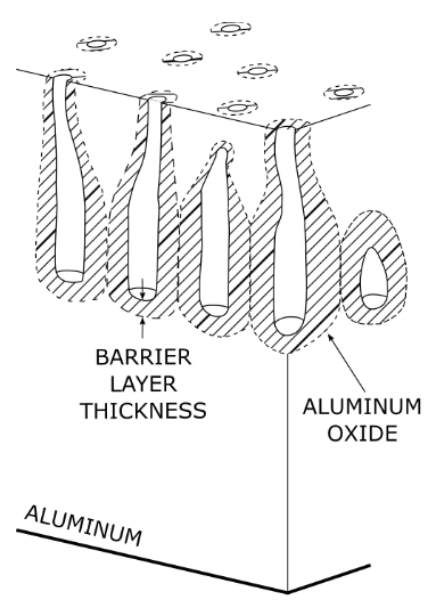

a) First Anodization

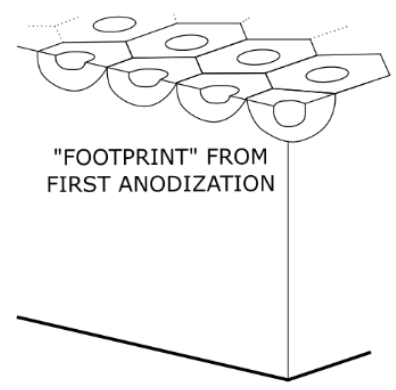

b) First anodization etch

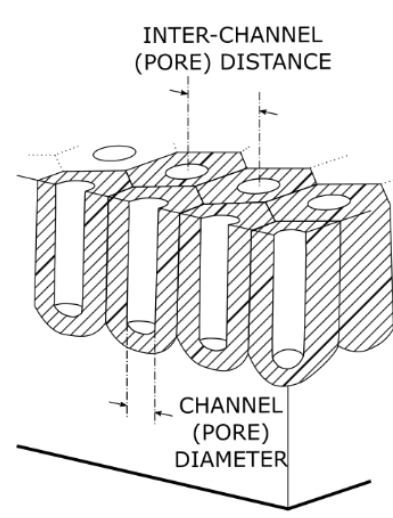

c) Second anodization

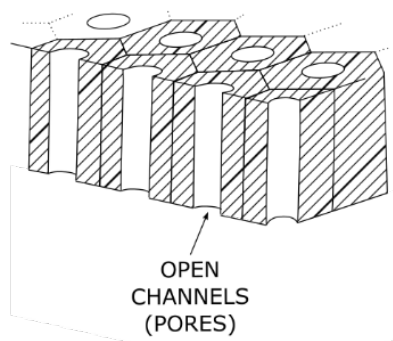

d) Aluminum and barrier layer etch

Fig. 2 Standard anodization procedure starting with high purity aluminum with a polished surface. The first stage corresponds to the formation of random channels near the surface which then self-organize (a). This alumina from the first anodization is then removed (b), leaving a footprint as starting point for a second anodization. The second anodization results in highly ordered nanochannels with a narrow size distribution (c). The backside of the aluminum is then removed and the channels opened creating pores that run across the entire thickness (d).

To attain higher homogeneity of the distribution of the channels, the electric field that results from the voltage applied through the cell must be uniform over the anodization area. To this end, the aluminum surface is smoothed prior to anodizing to avoid electric field concentration on protuberances by electrochemically etching in a 75:25 mixture of ethanol and perchloric acid, reducing its surface roughness from the micrometer to the nanometer range [52], [53]. After this, an optional pre-patterning is performed either by removing the alumina produced during a first anodization step [54] (Fig. 2b) and using the resulting "footprint" over the aluminum or by indenting the surface, for instance with a stamp [55]-[59]. Stamps also allow patterns different from cylindrical shapes, such as prisms, to be produced (see [47]). Starting from the prepatterned surface, the channels can then be grown with their length controlled precisely by the anodization time for the intended application (Fig. 2c). To obtain a porous template, the non-anodized aluminum at the back side and the barrier layer, formed at the end of each channel during anodization (Fig. 2c), are etched away (Fig. 2d). Variations of these stamp [60] and two-step [54] anodization procedures allow tailoring of the template's parameters and the NW diameter along its length.

As will be seen later, for certain applications like using arrays of NWs as microwave circulators, a higher order of the NWs in the array, obtained by either of these procedures, is a key factor for obtaining sharper circulation resonant frequencies and isolation. This means, the regularity of the order has an impact on the dipolar interactions of the NWs in the array and therefore on the device performance.

By using specific anodization potentials, at least two regimes of distinct channel diameters and inter-channel distances can be obtained for each electrolytic bath, known as mild (low-field) and hard (high-field) anodization. Whereas mild anodization potentials are directly applied and sustained, hard anodization potentials are generally ramped from the mild anodization ones. This is done to create a thick enough oxide layer that prevents the high fields from driving high local currents through the oxide layer, which would "burn" the surface [61]. Common diameters and inter-channel distance values for each bath and regime are summarized in TABLE I together with their respective anodization parameters. Different electrolytic baths' characteristics such as $\mathrm{pH}$, solubility or relative permittivity have an impact on pore diameter and inter-pore distance or can modify the effective electric field [48].

A quantity used to characterize the density of pores per unit area is the ratio of their (planar) surface area to the total surface area also known as porosity. In other words, it is the percentage of surface that is covered by pores. Assuming the pores are ordered in a hexagonal pattern, the porosity can be geometrically expressed as:

$$
P=\frac{\pi}{2 \sqrt{3}}\left(\frac{D_{p}}{D_{\text {int }}}\right)^{2} .
$$

Where $D_{p}$ is the pore diameter and $D_{\text {int }}$ is the inter-pore distance, illustrated in Fig. 2c (A detailed calculation can be found in section 1.2.2.5 of [46]). Typical values for each bath can be found in TABLE I, where two trends can be recognized: porosity lowers from mild to hard anodization regimes and increases as the potential is reduced throughout the baths. This is due to the controlled oxidation rates being dependent on the strength of the electric field as discussed before. 
TABLE II

THREE MAIN ANODIZATION BATHS WITH THEIR REPRESENTATIVE PORE DIAMETER AND INTER-PORE DISTANCE

\begin{tabular}{|c|c|c|c|c|c|c|}
\hline ANODIZATION BATH & Regime & Potential (V) & Pore Diameter (nm) & Inter-pore distance (nm) & Porosity (\%) & Reference \\
\hline \multirow{2}{*}{ Sulfuric Acid } & Mild & 25 & 30 & 60 & 22.7 & {$[65]-[67]$} \\
\hline & Hard & 70 & 50 & 130 & 13.4 & {$[68],[69]$} \\
\hline \multirow{2}{*}{ Oxalic Acid } & Mild & 40 & 35 & 110 & 9.2 & {$[66],[70],[71]$} \\
\hline & Hard & 140 & 60 & 300 & 3.6 & {$[60]$} \\
\hline Phosphoric Acid & - & 205 & 150 & 450 & 10.1 & {$[65],[72]$} \\
\hline
\end{tabular}

As the electric field driving anodization is controlled by a voltage supplied from a power source, it can be varied to change the oxidation rate and, therefore, create modulations of the channel diameter [60], [62]-[64]. An example of channel diameter modulation is shown in Fig. 3 where an electric current is applied (Fig. 3a), while the voltage is monitored. The process starts with mild anodization values (MA) setting the initial diameter, then, it is transitioned (TA) to hard anodization (HA) increasing the diameter to a maximum and finally returning to MA "resetting" the diameter. The resulting shape of the channel (Fig. 3b) follows the current profile (Fig. 3a) and shows that controlled diameter modulations can be achieved along the channel. This fact has been exploited to tune the optical properties of the template [63], [73] and is envisioned to be used in coatings [74] and steam generation [75], for example. For more information on applications of alumina templates, the reader is referred to the excellent reviews by Lee [48] and Sulka [47].

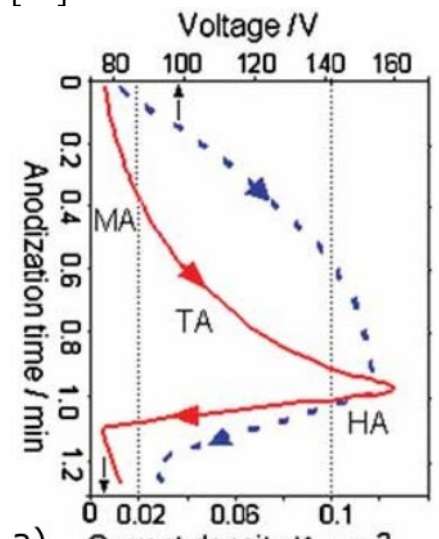

a) Current density $/ \mathrm{A} \mathrm{cm}^{-2}$

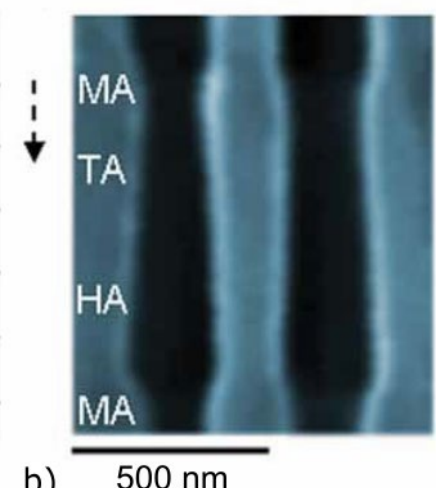

Fig. 3 Example of channel diameter modulation during anodization (figure adapted from [63]). This galvanostatic cycle (a) starts at mild anodization currents (MA), then is ramped up, transitioning (TA) to hard anodization (HA) and then returned to MA. The resulting channel shape is shown in (b).

It is worth mentioning that some constrains and advantages of the methods described so far. When performing indentation to pre-pattern the surface, the output area is limited by the area of the stamp or the area accessible by the milling tool in a reasonable (cost-effective) time. In addition to this, the indentations cannot deviate significantly from the self-ordered inter-pore distance obtained for each of the conditions summarized in TABLE I. Also, although in principle the freestanding template could be a few microns thick, it is commonly fabricated to be around $60 \mu \mathrm{m}$. This is because, without a mechanical support, thinner templates are easily broken when handling them even when a frame of aluminum is kept around the anodized part. On the other hand, once anodized, the channel diameters in each template can be enlarged up to around $80 \%$ of the inter-channel distance by submerging in acidic baths [46], [76], limiting but also allowing the tailoring of porosity for specific properties. Additional steps must be performed on alumina templates to prepare them for the electrochemical growth of the NWs. One option is the deposition of a conductive thin film on one side of the porous template, shown in Fig. 2d, that serves as a working electrode for electroplating. In the case when such conductive film is grown in a way where it does not to fully cover the pores, the formation of nanotubes is favored (Fig. $\mathbf{4 b}$ ). A different route is to start reducing the thickness of the barrier layer after the second anodization, as shown in Fig. 2c by progressively lowering the applied bias voltage to a small value; thus, creating "dendrites" at the end of each channel. These dendrites allow the flow of current into the aluminum for sufficiently high applied potentials, using a pulsed electrodeposition method explained below, and thus eliminate the need to remove the non-anodized aluminum as in Fig. 2d.

The combination of diameters and inter-channel distances, in conjunction with the possibility of widening and modulating the diameters, allows the creation of virtually any shape within those constrains. This implies that NWs grown inside the templates can also have, in principle, any shape within those constrains.

\section{B. Electroplating of cylindrical nanowires}

Electroplating of metals is used to get coatings on surfaces in several areas of industry and research [77], [78]. It consists of the reduction of metal ions from an electrolyte at a working electrode with specific conditions of temperature, $\mathrm{pH}$, concentration and applied electrical bias in an electrolytic cell. Electrolytes are prepared commonly from metal hydrates in aqueous solutions, where they dissociate into an ionized metal cation and a polyatomic anion, and using chemical buffers to maintain the $\mathrm{pH}$ value during plating. These ions, of radii of about hundred picometers, migrate to the working electrode, due to the electric field, where they are reduced at the negatively charged surface. This allows electroplating to be performed in nano-sized electrode areas, such as those inside of the channels 
of alumina templates, causing the deposited metal to acquire their shape while it grows for several tens of microns.

Most (if not all) of the electrolytes used in nanofabrication of NWs have their origin in industrial recipes and can be found in technical texts [77]. As a general rule, if an electrolyte has been used in industrial applications, it can be used for nanotechnology research. For instance, nickel electroplating is carried out with variations of the well-known "Watts" solution [79], [80], and other well-known electrolytes exist for copper [81], gold [82] and iron [83]. Nevertheless, research is carried out to improve or produce new electrolytes for nanotechnology applications [84]-[86]. For example, a single electrolyte can contain two different metallic species with different reduction potentials and can be used to deposit alternating metallic layers [33], [35], [87]. Also, intrinsic material properties are sought to be controlled while plating: crystal anisotropy can be controlled by modifying deposition $\mathrm{pH}$ [50], [86], [88], [89] and magnetic exchange interaction can be tailored by depositing alloys [83], [90]-[94]. One of the most desired property is single crystalline deposition, which has been achieved to a certain degree [95], [96]. These techniques are continuously being adapted and improved to obtain the needed industrial or research driven material properties.

There are mainly three methods to control the electroplated metal growth: potentiostatic, galvanostatic and pulsed electrodepositions. In the potentiostatic method, a constant voltage relative to a reference electrode is applied across the electrolytic cell. In the galvanostatic method, the current is set and the source adjusts the output voltage so as to keep that current constant with a feedback loop. In both cases, a potential high enough for the metal ions to overcome the naturally formed electrical double layer and reduce at the working electrode needs to be applied [97]. The pulsed electrodeposition method is mostly used in membranes with dendrites [98], and consist of two current pulses and a recovery time of amplitude and width depending on the electrolyte used. A first pulse deposits the material but charges the barrier layer increasing the thickness of the electrical double layer like a capacitor, which is discharged with a second inverted pulse of the same amplitude of the lowest voltage used to create the dendrites. Then a recovery time, when no potential is applied, is given to replenish the ion concentration and balance the $\mathrm{pH}$ around the working electrode so the process can be restarted. As metal ions are reduced at the working electrode by charge transfer and side reactions take place at the counter $(\mathrm{Pt})$ electrode, in all methods the measured (or controlled) current is an indicator of the rate of deposition. The selection of each method depends on the template available, power source, control system, quantity and quality of the deposited products.

TABLE II summarizes some of the most common magnetic materials electroplated into alumina templates. It is not intended to be complete nor to include all references (only one reference per material is used) but to serve as a starting point and as example of the range of possible NW materials that can be grown using this method. In addition to this, doping can be achieved as for example Pt-doped iron [99] and annealing leads to metal oxides (see for example the end of section III and sections IV and V.A below).

TABLE III

MAGNETIC MATERIALS COMMONLY ELECTROPLATED INTO ALUMINA TEMPLATES

\begin{tabular}{|c|c|c|c|}
\hline Material & $\begin{array}{c}\text { Common } \\
\text { electrolyte reagents }\end{array}$ & Comment & Reference \\
\hline $\begin{array}{l}\text { Nickel } \\
(\mathrm{Ni})\end{array}$ & $\begin{array}{l}\text { Nickel Sulfate } \\
\text { Nickel Chloride }\end{array}$ & Elemental & [100] \\
\hline $\begin{array}{l}\text { Cobalt } \\
(\mathrm{Co})\end{array}$ & $\begin{array}{l}\text { Cobalt Sulfate } \\
\text { Cobalt Chloride }\end{array}$ & Elemental & {$[95]$} \\
\hline $\mathrm{CoNi}$ & $\begin{array}{c}\text { Mixture of } \\
\text { elemental electrolytes }\end{array}$ & Alloy & {$[90]$} \\
\hline $\mathrm{Co} / \mathrm{Ni}$ & $\begin{array}{l}\text { Interchange of } \\
\text { elemental electrolytes }\end{array}$ & $\begin{array}{c}\text { Multi- } \\
\text { segmented }\end{array}$ & {$[36]$} \\
\hline CoPt & $\begin{array}{c}\text { Mixture of } \\
\text { elemental electrolytes }\end{array}$ & Alloy & [101] \\
\hline Iron $(\mathrm{Fe})$ & Iron Sulphate & Elemental & [102] \\
\hline $\begin{array}{l}\text { Galfenol } \\
(\mathrm{FeGa})\end{array}$ & $\begin{array}{l}\text { Iron Sulfate } \\
\text { Gallium Sulfate }\end{array}$ & Alloy & [45] \\
\hline $\begin{array}{l}\text { Permalloy } \\
(\mathrm{FeNi})\end{array}$ & $\begin{array}{c}\text { Mixture of } \\
\text { elemental electrolytes }\end{array}$ & Alloy & [103] \\
\hline $\mathrm{FeCo}$ & $\begin{array}{c}\text { Mixture of } \\
\text { elemental electrolytes }\end{array}$ & Alloy & [104] \\
\hline $\mathrm{FeNiCo}$ & $\begin{array}{c}\text { Mixture of } \\
\text { elemental electrolytes }\end{array}$ & Alloy & [105] \\
\hline $\mathrm{FeCoCu}$ & $\begin{array}{c}\text { Mixture of } \\
\text { elemental electrolytes }\end{array}$ & Alloy & [106] \\
\hline FePt & $\begin{array}{c}\text { Mixture of } \\
\text { elemental electrolytes }\end{array}$ & Alloy & [107] \\
\hline
\end{tabular}

In summary, with the described methods, a wide range of NWs with different shapes and intrinsic material properties can be fabricated (Fig. 4a-d). Control of the shape anisotropy and demagnetization energy can be achieved by depositing NWs into templates modulated in diameter Fig. 4c [64], [91]-[93], [108] and controlling their length either by monitoring the total deposited charge (total length of the NW) or by introducing nonmagnetic segments between magnetic segments and monitoring each segments' length (Fig. 4d) [45], [94], [109][112]. Composition modulations can also be performed by selecting the deposited metal ions by manually substituting the electrolyte [36], [90] and it is even possible to match diameter and composition modulations [113]. 


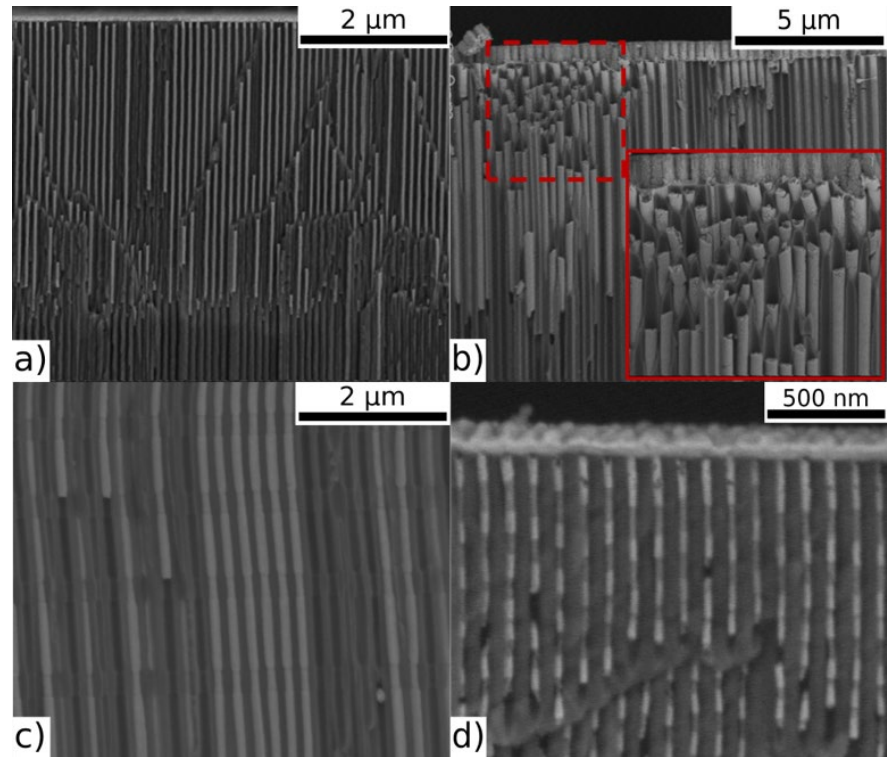

Fig. 4 SEM imaging of (a) Fe90Co10 NWs with diameter (D) of $60 \mathrm{~nm}$, (b) Fe30Co 70 nanotubes with $\mathrm{D}=200 \mathrm{~nm}$, (c) $\mathrm{Fe} 28 \mathrm{Co6} 7 \mathrm{Cu} 5$ diameter-modulated NWs with D1 $=120 \mathrm{~nm}$ and D2 $=140 \mathrm{~nm}$, (d) Co/Pt composition-modulated or multisegmented NWs with $\mathrm{D}=40 \mathrm{~nm}$.

\section{DC APPLICATIONS - PERMANENT NANO-MAGNETS AND ELECTRODES}

Whether isolated or collectively, NWs are used as a static source of magnetic fields for different applications. To this end, it is crucial that they are designed with large remanence and coercivity (e.g., high energy product $B \cdot H$ ) to produce large stray fields and prevent demagnetization, respectively. In highly packed nanowire arrays, the dipolar interaction between neighboring NWs produces an effective demagnetization that reduces the energy product [114], [115]. To overcome this effect the magnetocrystalline anisotropy energy can be tailored to keep the easy axis parallel to the NW's long axis. In this regard, transition metal alloys such as $\mathrm{Co}_{\mathrm{x}} \mathrm{Pt}_{1-\mathrm{x}}$ or $\mathrm{Fe}_{\mathrm{x}} \mathrm{Pt}_{1-\mathrm{x}} \mathrm{NWs}$ constitute a good example, due to their controllable and large magnetocrystalline anisotropy $\left(\mathrm{K}_{\mathrm{u}} \approx 5 \times 10^{6} \mathrm{~J} / \mathrm{m}^{3}\right)$ [101], [107]. Alternative magnetic alloys, which do not contain rare-earth elements or Pt, such as FeCo, are also considered, due to their high Curie temperature and large saturation magnetization.

Several attempts have been made to promote a magnetic hardening (increased coercivity) by adjusting the alloy composition, adding a non-magnetic material into the system or by thermal annealing [116]-[119]. As an example of the latter, a significant increase in coercivity and magnetocrystalline anisotropy energy was observed in $\mathrm{Fe}_{30} \mathrm{Co}_{70}$ NWs, embedded in alumina templates, by adding 5 at. $\% \mathrm{Cu}$, and performing a subsequent thermal treatment. Saturation magnetization, measured under $2 \mathrm{~T}$ applied field, resulted in values of about 2.0 $\mathrm{T}$ and $1.7 \mathrm{~T}$ for as-deposited and $500{ }^{\circ} \mathrm{C}$ annealed samples, respectively. After annealing, an increase in coercivity, which increased the $\mathrm{B} \cdot \mathrm{H}$ energy product, was observed in both, $\mathrm{FeCo}$ and $\mathrm{FeCoCu}$ samples, but more effectively in the samples containing $\mathrm{Cu}$ (Fig. 5a).
The large coercivity of thermally treated Cu-doped NWs is attributed to the increase in magnetocrystalline anisotropy energy as a result of $b c c$ lattice strain and changes in the NW microstructure [115], [119].
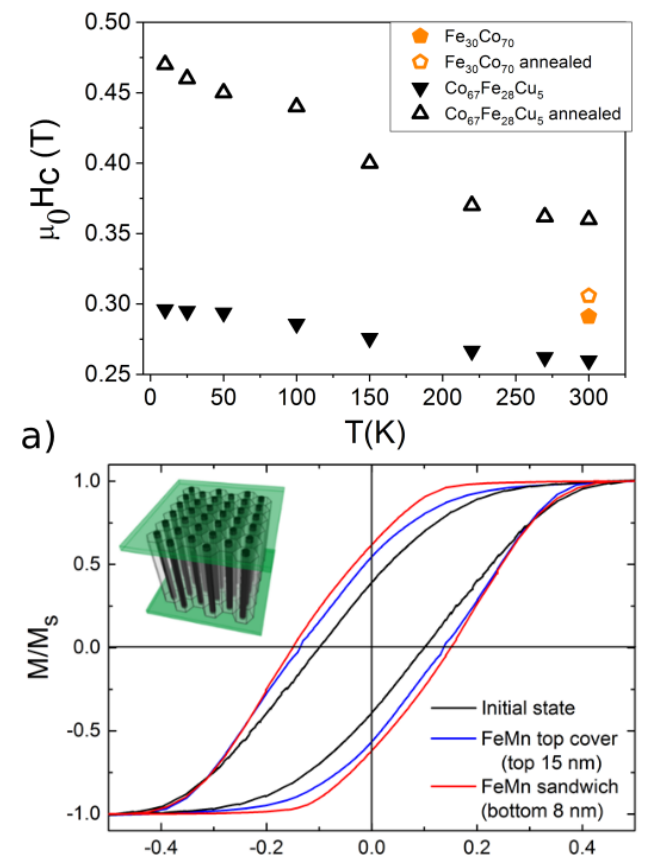

b)

$B(T)$

Fig. 5 (a) Low temperature dependence of coercive field of FeCo (orange symbols) and $\mathrm{Cu}$ - doped FeCo (black symbols) NWs, with $20 \mathrm{~nm}$ in diameter, before and after annealing at $500{ }^{\circ} \mathrm{C}$ (figure adapted from [119]), (b) Magnetic hysteresis loops of $40 \mathrm{~nm}$ diameter $\mathrm{Fe}_{30} \mathrm{Co}_{70} \mathrm{NW}$ arrays at $300 \mathrm{~K}$ for 3 different states: as-prepared, the top-tips of the NWs covered with FeMn and both (top and bottom) ends covered with FeMn. The inset shows the schematic view of the sandwiched AFM/FM/AFM structure (figure adapted from [104] (C) IOP Publishing. Reproduced with permission. All rights reserved).

A different approach for improving the magnetic hardness of NWs consists in exploiting the interfacial exchange between ferromagnetic-antiferromagnetic (FM-AFM) or between ferromagnetic materials with different and high magnetocrystalline anisotropy [120]. As the NWs demagnetize via nucleation from the NW ends or de-pining, any way of stalling this effectively increases the coercive field [104]. One way to achieve this starts by etching the top and bottom layer of alumina, resulting in free-standing FeCo tips sticking out of the template. The free tips are cover by a thin AFM $\left(\mathrm{Fe}_{50} \mathrm{Mn}_{50}\right)$ layer creating an $\operatorname{AFM}(10 \mathrm{~nm}) / \operatorname{FM}(16 \mu \mathrm{m}) / \operatorname{AFM}(10 \mathrm{~nm})$ structure (Fig. 5b-inset). The magnetic measurements (Fig. 5b) revealed that this sandwiched AFM/FM/AFM structure shows a significant magnetic hardening $(50 \%$ increase in coercivity and $63 \%$ in remanence) with a doubled energy product at room temperature [104].

The stray field emanating from the tips of the NWs in an array depends on their magnetic properties and interactions as well. These interactions are commonly investigated by studying their first order reversal curves (FORC) diagrams [121]-[123] or by directly imaging their fields with TEM tools [12]. For 
example, in iron, nickel and cobalt NWs of $50 \mathrm{~nm}$ diameter, these stray fields are highly localized and decay rapidly (below $200 \mathrm{~nm}$ ) with the distance from the tip [12].

Although isolated NWs fabricated inside alumina templates are envisioned to work as MFM tips, to our knowledge, not many reports have appeared, since Yang et al attached single NWs dispersed in water to a cantilever tip using a dielectrophoresis process [15] and Downey et al used a nanomanipulator to pick them from a stripped template [124] mainly to test their mechanical properties (see Fig. 6a).

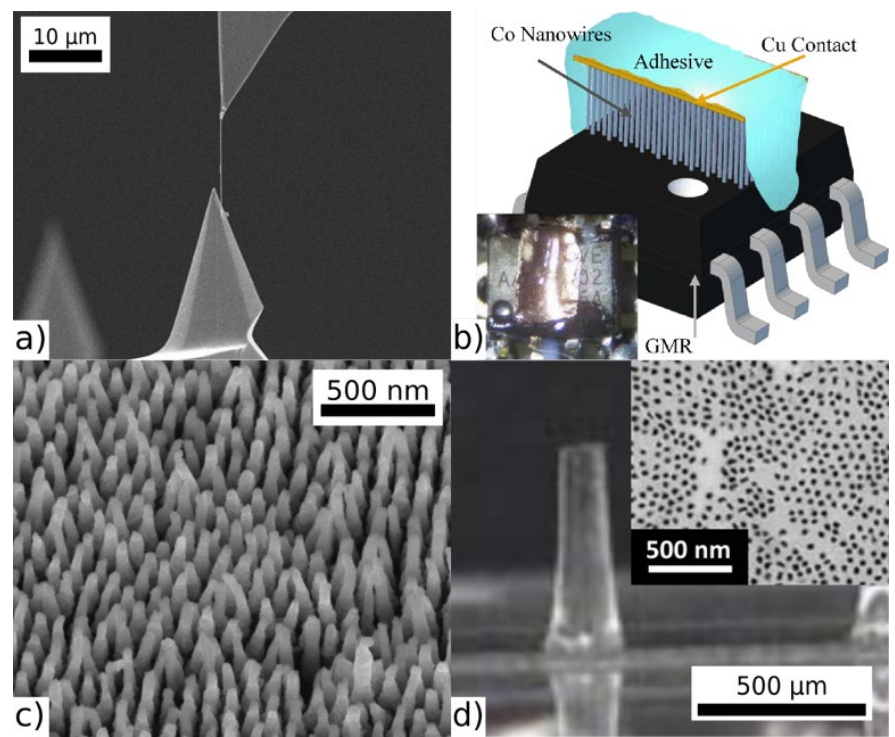

Fig. 6 Cylindrical nanowires (a) attached to an atomic force microscope tip (Reprinted from [124], with the permission of AIP Publishing.), (b) as vibration sensor (reprinted from [125] (C) 2013 IEEE), (c) as hematite electrode for water splitting (Reprinted with permission from [126] Copyright 2019 American Chemical Society) and (d) inside a polymer micropillar for force sensing (Reprinted from [127] (C) 2015 IEEE) (the inset shows the NWs aligned and embedded inside the polymer).

Free standing arrays of bare NWs have been used as cilia for liquids/gas flows [125], acoustic [128] and pressure [129] sensors by placing them in close proximity to the surface of a GMR sensor, as can be seen in Fig. 6b. A limiting factor in those devices is the NW corrosion as well as the fact that the shape anisotropy energy is way larger than the energy changes resulting from the small mechanical deflections. This means that the deflections do not modify greatly the stray magnetic fields, limiting the sensitivity. One way to overcome these limitations is to release the NWs from the array and embed them into a polydimethylsiloxane (PDMS) matrix to create cilia-like structures like the one shown in Fig. 6d [127], [130]. The PDMS structure protects the NWs from corrosion and provides higher mechanical flexibility, allowing more pronounced deflections. This allowed to fabricate force sensors for flow, acoustic, pressure or robotic applications [13], [14], [131][133] and energy harvesters [16], [17].

Arrays of NWs and nanotubes have been used as electrodes for multiple applications due to their high surface area. Electrodes of annealed iron, with and without doping, produce the "hematite" iron-oxide phase known to perform water oxidation in photo-electro-chemical water splitting as shown in Fig. 6c [126], [134]-[136]. On the other hand, several other materials are being grown in alumina templates to produce batteries, supercapacitors and/or fuel cells [137] and have been considered to work as thermoelectric generators [138].

\section{LOW FREQUENCY APPLICATIONS - BIOMEDICINE}

Magnetic NWs are being used for various biomedical applications benefiting from the possibility to manipulate them with light [23], [139] or external magnetic fields [22], and sense them with magnetic field sensors (see section III). The applied magnetic field frequencies range from $0.1 \mathrm{~Hz}$ external magnetic fields for stem cell scaffolds over $100 \mathrm{~s}$ of $\mathrm{kHz}$ for hyperthermia. When in the presence of air, transition metal nanostructures naturally develop an oxide layer at their surface due to oxygen inward diffusion [26], [140], [141] (See Fig. 7c). This oxide layer makes them biocompatible [142] and biodegradable [143], [144] to the point that they are approved for in vivo use in humans [145]. This oxide layer can be thickened by annealing [26] and functionalized to enhance colloidal stability [146], target certain cells [25], [102], [146] and label them [147]. Since the aspect ratio of NWs can be tailored at the fabrication step, they can take shapes from the semisphere/cube to the nanowire geometries. High aspect ratio NWs facilitate them to behave as magnetic dipoles with which it is more efficient to perform cell separation with static applied magnetic fields [148], [149].

Ferromagnetic NWs are internalized by living cells as was shown in early in vitro studies [150]-[152] without being toxic to the cells (low cytotoxicity) even for high concentrations ( $10000 \mathrm{NWs}$ per cell) or long incubation times (5 days). Furthermore, some cells are able to degrade NWs, reducing them to small aggregates and distributing them to neighboring cells [153] possibly due to their polycrystallinity and composite structure [154]. The internalization process takes different times depending on the cells and the NWs, yet once inside, the cells can even divide without problem, as was shown with a machine-learning-assisted sorting of NWs in endosomes [152].

\section{A. Drug delivery}

The capability for drug delivery using NWs depends on drug load, cell targeting and controlled release. Low frequency $(\mathrm{Hz})$ external magnetic fields can manipulate single ferromagnetic NWs by producing a torque that induces rotation due to their high aspect ratio. This can be used to propel and push, pull or rotate cellular and sub-cellular objects [155], [156], as shown in Fig. 7a, b. Using a rotating in-plane magnetic field together with a static one perpendicular to the plane, NWs made of $\mathrm{Ni}$ and flexible silver have been shown to propel, pick up drugloaded magnetic microparticles and deliver them through a microchannel to living cells [157]. In addition to this way of loading, antibodies can be coated on the naturally formed oxide layer of NWs (Fig. 7c) by functionalization to perform intracellular delivery (Fig. 7d) [149], [158], [159]. 


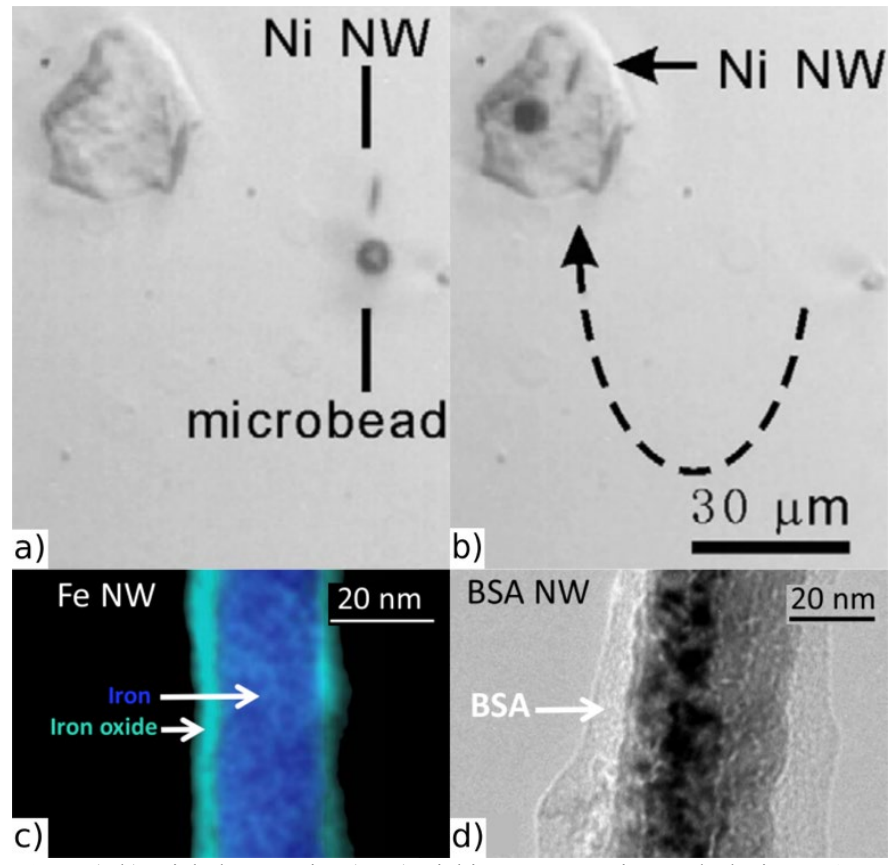

Fig. 7 (a,b) Nickel nanowire (NW) picking, transporting and placing a $6 \mu \mathrm{m}$ diameter polysilicon microbead on a epidermal cell (Reprinted from [155] Copyright 2012, with permission from Elsevier). (c) Electron energy loss spectroscopy image of an iron nanowire displaying its oxide layer and (d) transmission electron microscopy image of an iron nanowire coated with bovine serum albumin (BSA) (Figures c and d adapted from [158] (C) 2019 IEEE).

NWs can also serve as remote triggers for other drug-carriers to release the drug. Poly (N-isopropylacrylamide) (PNIPAM) is a micro-gel that swells below a certain temperature and shrinks at a higher temperature, thereby it absorbs and expels a solvent (drug), respectively [160]. When NWs are embedded into PNIPAM, an external magnetic field ( $\mathrm{kHz}$ frequency range) causes them to vibrate resulting in friction (in addition to possible hysteresis losses) and therefore heating, triggering the release of the solvent. With this method, for example, Rhodamine (B) was loaded into PNIPAM, transported through a microfluidic channel and released in portions into living cells using a pulsed magnetic field of $1 \mathrm{mT}$ and $20 \mathrm{kHz}$ [161]. The ability to release multiple drug doses from the same PNIPAM carrier by repeated application of the magnetic field is an additional feature of this method.

\section{B. Magnetic NWs for cancer treatment}

There are mainly three methods (which can be combined) to destroy cancer cells once the NWs have targeted and possibly have been internalized by them: release specific drugs, induce heat or mechanically affect the cell's internal structure. The models for the mechanical heating of magnetic nanoparticles in liquids using external AC magnetic fields have been widely studied [162], [163]. When an AC (kHz) field is applied to NWs internalized by cancer cells, their hysteresis losses makes them act as localized heat sources that induces their death (hyperthermia) [164] as they are especially sensitive to temperature changes [165]. Both the applied magnetic fields and magnetic properties of NWs play an important role in determining the amount of heat released, which is characterized by the specific absorption rate (SAR) [22]. This SAR can be experimentally quantified by inspecting the hysteresis and minor loops of NWs [166] and relating them with calorimetric studies [167], [168].

Mechanical stress can be induced in living cells using internalized NWs and applying external magnetic fields at low frequencies. Rotating NWs at low frequencies can deform the cytoplasm and cell nucleus [169] or produce cell membrane rupture [4] to cause cell death. For instance, internalized Ni NWs induced cell deaths of fibroblasts in a $240 \mathrm{mT}$ rotating field at $1 \mathrm{~Hz}$ [170], colon cancer cells in a $0.5 \mathrm{mT}$ field at $1 \mathrm{~Hz}$ [4] or kidney cells at frequencies from around 2 to $11 \mathrm{~Hz}$ [171]. These small field and low frequency values make the method particularly attractive from an energy and instrument point of view, which can be kept low and compact, respectively.

Combining several treatment methods into one platform has been shown to increase the efficacy of cancer cell killing. An example of combined drug-release and mechanical actuation on cells was performed with Fe NWs loaded with doxorubicin, targeting breast cancer cells and actuating them with a $1 \mathrm{mT}$ magnetic field at $10 \mathrm{~Hz}$ [102]. Recently, those two methods were further combined with photothermal therapy, induced by a near infrared laser. Using an iron/iron oxide core/shell NW, a very high photothermal efficiency of around $80 \%$ was achieved and the combinatorial treatment resulted in nearly complete cancer cell eradication [139].

\section{Magnetic Resonance Imaging (MRI) contrast agents}

In magnetic resonance imaging (MRI) high magnetic fields align, to some extent, the spins of protons from hydrogen atoms (mostly in water molecules). An additional radiofrequency (RF) pulse then excites those aligned spins and the energy released as they re-align with the field is sensed. In different types of tissues, the energy released and the time it takes for the protons' spins to realign (relaxation time) is different, allowing their differentiation. When the static magnetic field is applied, spins precess in phase around it with a frequency known as Larmor frequency, which depends on the magnitude of the applied field. After the RF pulse is applied at 90 or 120 degrees with respect to the static field, the spins relax back to precess around the static field with Larmor frequency in a time $T_{1}$. Nevertheless, some spins remain out of phase longer due to spin-spin interactions, and relax with a higher relaxation time $\mathrm{T}_{2}$.

Contrast agents are sometimes used to reduce the relaxation times $T_{1}$ and $T_{2}$ : the faster they realign, the brighter the image [172]. This is done by creating a local magnetic field, which enhances the proton spins re-alignment, meaning that contrast agents are not directly visualized but they only affect their surroundings. This also explains why most paramagnetic contrast agents shorten $T_{1}$, while superparamagnetic and ferromagnetic shorten mostly $\mathrm{T}_{2}[173]$. The performance of a 
magnetic nanostructure as a contrast agent is measured via its relaxivity $\left(\mathrm{r}_{\mathrm{i}}=1 / \mathrm{T}_{\mathrm{i}}\right)$, which is a measure of the relaxation rate and depends on the concentration of the magnetic agent [172]. Then, contrast agents can be used for positive $\left(T_{1}, r_{2} / r_{1}<5\right)$ or negative $\left(\mathrm{T}_{2}, \mathrm{r}_{2} / \mathrm{r}_{1}>8\right)$ based images [174] according to their magnetic properties, geometry and coating [175].

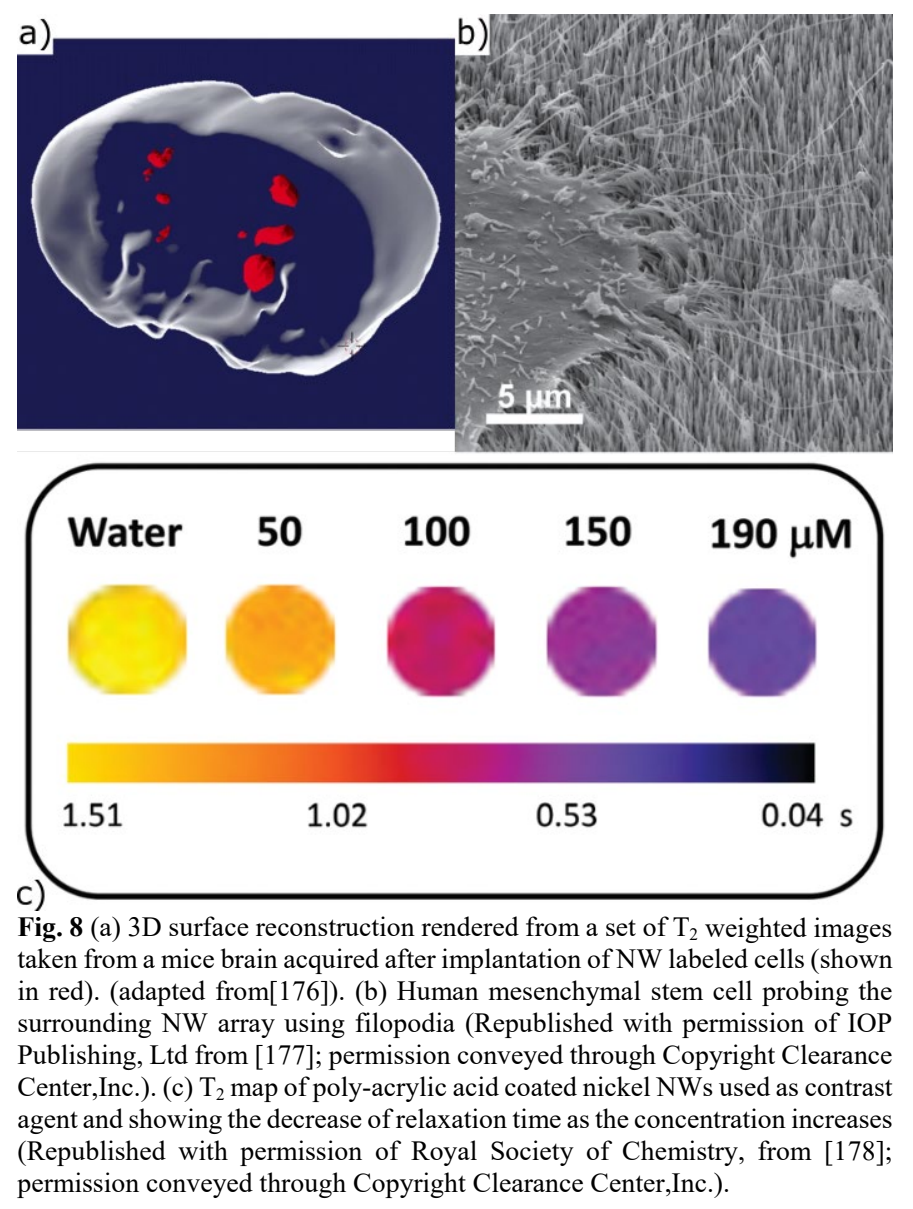

Ferromagnetic NWs with high aspect ratios, which can create large local magnetic field inhomogeneities, can be employed as $\mathrm{T}_{2}$ contrast agents [179] and have been tested in-vivo as seen in Fig. 8a. This has been used, for example, in Fe and Fe-Au NWs, where higher aspect ratios increased $r_{2}$ and coating of the $\mathrm{Au}$ segments increased $r_{2}$ without changing $r_{1}$ [27]. Nickel NWs have also been used as $T_{2}$ contrast agents at $1.41 \mathrm{~T}$ with $\mathrm{r}_{2}$ values comparable to commercially available contrast agents based on iron-oxide nanoparticles [178]. Their $\mathrm{T}_{2}$ contrast map measured at $3 \mathrm{~T}$ is shown in Fig. 8c, where faster relaxation times are found for high concentrations.

\section{Cell scaffolds $\mathrm{NW}$}

Stem cells research is of growing interest, due to the potential for new treatments of different diseases and conditions. For example, in tissue engineering, mesenchymal stem cells (MSC) can differentiate (change from one cell type to another) into osteoblasts and generate bone tissue, which could assist the regeneration of patients with bone-degenerative diseases [180], [181]. The properties of the microenvironment and the matrix which the cell is embedded in, were shown to have a direct influence on the cell and its fate, i.e. the type of cell it is going to differentiate into [182], [183]. Consequently, research on stem cell scaffolds has focused on nano-engineering the matrix stiffness and geometry. Studies have been performed, for example, on polymeric nanofibers [184], nano-pits [185], $\mathrm{TiO}_{2}$ nanotubes [186], hydroxyapatite nanorods [187] and silicon NWs [188].

By releasing magnetic NWs only partly from the alumina template, nano-surfaces densely packed with free standing NWs can be created. Benefiting from the control over geometrical parameters provided by this fabrication method, stem cell scaffolds with different NW diameters can be realized. They mimic different environmental stiffness's, which result in different cell responses. In recent studies, MSCs on ferromagnetic NWs exhibited a contracted shape, shown by the clustering of the focal adhesion vinculin protein [177] (see Fig. 8b), which is linked to osteogenesis [188]. An advantage of these ferromagnetic NWs scaffolds over other materials is that they could be manipulated by external magnetic fields, providing interesting opportunities for mechanical cell stimulation on the nanometer scale. On the other hand, arrays of Fe-Au NWs have been used as plasmonic scaffolds to enhance surface enhanced Raman scattering signals [76]. Thereby, small gaps between the NWs is required to obtain a field enhancement effect. NW separations of $<10 \mathrm{~nm}$ were achieved by widening the pores of the alumina template before electrodeposition.

\section{HIGH FREQUENCY - ELECTRONIC ELEMENTS}

\section{A. Non-volatile Data Storage}

Although arrays of NWs have been proposed to be used as memory devices taking advantage of their high internal interaction field [3], most of the envisioned data storage applications require bits to reside within individual NWs.

Two main types of 3-dimensional magnetic memories using NWs are under research: memristors [189]-[191] and the cylindrical analog of the "racetrack memory" [192]. The former targets NAND flash working in the microseconds $(\mathrm{MHz})$ regime and the latter targets DRAM working at the nanoseconds regime $(\mathrm{GHz})$ [193]. In NWs memristors, one of the most common resistive switching mechanisms consists in applying an electric field which produces point defects migration, creating conductive paths through the oxide layer in a metal/oxide/metal capacitor structure and reducing its resistance (logical 0) [194]. These paths remain after the electric field that created them has been removed and shrink with the application of heat or another electric field of inverse polarity, returning the structure to a high resistance state (logical 1). This type of memory has been reported in homogeneous $\mathrm{NiO}$ [195], multisegmented $\mathrm{Au} / \mathrm{NiO} / \mathrm{Au}$ [196], 
$\mathrm{Pt} / \mathrm{NiO} / \mathrm{Pt}$ [197], $\mathrm{Ni} / \mathrm{CuO}_{\mathrm{x}} / \mathrm{Ni}$ [198], $\mathrm{Ni} / \mathrm{NiO} / \mathrm{Ni}$ [199], coreshell $\mathrm{Ni} / \mathrm{NiO}$ [200] and $\mathrm{TiN} / \mathrm{TiO}_{2} / \mathrm{Pt}$ [201] NWs amongst others. A thorough compilation of structures and measurement techniques can be found in [190].

Due to their high shape anisotropy, NWs typically represent one dimensional magnets with their magnetization pointing parallel or antiparallel to their long axis (up or down in Fig. 9a). This could be exploited for data storage, where parallel magnetization corresponds to logic 0 and antiparallel to logical 1. In order to realize a multi-bit 3D memory, several bits need to be stored along the NWs, as shown in Fig. 9b, requiring multi-domain states. This can be achieved by creating DW pinning sites along multisegmented NWs via compositional changes [202] or diameter modulations [37], [203]. Each segment's magnetization direction reverses either by coherent rotation or by nucleation and propagation of a DW, depending on their composition (e.g., defining its magnetocrystalline anisotropy) and length [111], [204]. This reversal can be driven by magnetic fields [36], [37], electric currents [43], [205] or a combination of both [44] with DWs possibly transforming during motion [206]. In particular, the experiments with cobalt and nickel-based NWs of diameters around $90 \mathrm{~nm}$ have resulted in current-induced DW velocities up to $600 \mathrm{~m} / \mathrm{s}$ [44], [205].

\section{$\square$ Ferromagnet \\ Ferromagnet \\ Barrier \\ $\Longrightarrow$ Reference \\ $\Longleftrightarrow$ SAF \\ $\triangle \nabla \begin{aligned} & \text { Magnetization } \\ & \text { direction }\end{aligned}$}

a)

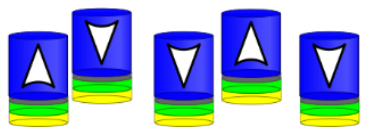

b)

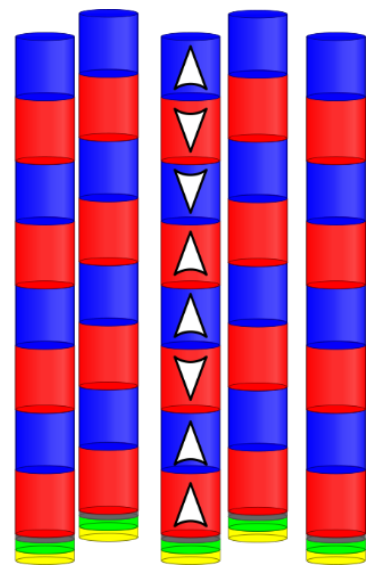

Fig. 9 Magnetic memory designs based on cylindrical NWs. (a) Perpendicular anisotropy magnetic tunnel junction memory: one bit can be stored in the magnetization state of each junction. (b) Multisegmented NWs with several bits stored in the magnetization state of each segment.

One of the main challenges of these perpendicular anisotropy memories is the stray field of each memory element that creates interference as the element density increases and they are packed closer together. This effect has been widely studied for NWs [103], [108], [122], [207], [208] and could now be useful for perpendicular anisotropy magnetic tunnel junction (p-MTJ) based memories [209], [210] as their packing density increases. In these p-MTJs, a thick ferromagnetic (storage) layer is deposited on top of an MTJ so that its thickness is in the order of or larger than the diameter of the MTJ [209], [211] or the thickness of the free layer of a MTJ is increased until the shape anisotropy dominates [210]. This combination of perpendicular shape anisotropy and interfacial perpendicular magnetic anisotropy increases the device's thermal stability, i.e., the minimum energy required to switch the memory between two states well above room temperature activation. A cylindrical 3D "racetrack memory" could be considered an extension of pMTJs, which are used in magnetic random access memories (MRAM) [209], [210], and are produced by companies like Toshiba, Samsung and Everspin-Global Foundries, among others [193].

\section{B. Microwave electronics}

Arrays of ferromagnetic NWs absorb microwaves at tunable resonant frequencies depending on the deposited magnetic material (or materials), their geometry, membrane porosity and an optional applied external field [212]-[214]. This absorption occurs during the ferromagnetic resonance (FMR) when the applied microwave frequencies coincide with the frequency at which magnetic moments precess about the internal effective magnetic field. This occurs dynamically: the microwave magnetic field knocks the magnetic moments away from the internal effective magnetic field and they precess about this latter as they re-align. Resonance occurs, then, when the microwave magnetic field reinforces the precession at the same frequency. The internal effective magnetic field depends on the magnetic energy terms, which can be tailored, as discussed in section II, namely exchange, crystal and shape anisotropy and magnetostatic interactions. Through this mechanism, most of the microwave is absorbed by the NWs, which then act as tunable filters.

A common approach to fabricate microwave devices is using the NWs embedded in the template as a substrate in a microstrip as can be seen in Fig. 10a. This microstrip consists of a conducting line separated by the substrate from a ground plane, the whole of it being a transmission line. In addition of being a dielectric, this substrate has magnetic properties which are appreciable thanks to the NWs diameters being smaller than their skin depth at high frequencies [212]. These magnetic properties contribute to the majority of the losses when a microwave is passed through the transmission line due to the FMR explained before [212], [214]-[216]. Vector network analyzers are used to generate and route these microwaves into the NWs microstrip through (commonly) two ports and characterize their scattering parameters (S). These scattering parameters are related to both the amplitude and phase shift $(\varphi)$ properties of the scattered waves at the microstrip and quantify the power losses.

The frequency response of the transmission scattering coefficient $\mathrm{S}_{21}$ (forward from port 1 to port 2) for CoFe NWs, both measured (black) and simulated (gray), is shown in Fig. 10b [214]. The strong absorption dips are related to the FMR and are present even at zero applied field, as explained before. The DC magnetic field applied along the long axis of the NWs modifies the precession frequency of the magnetic moments as they re-align increasing the frequency needed to reach 
resonance.

Clever, slight modifications to the growth methods explained in section II.B allow the production of microstrips where the NWs height changes along the conducting line width [217], [218]. In other words, the effective permittivity of the substrate varies due to the change in absorption depth along the width. This asymmetry causes the microstrip to behave differently in forward (+) and backward (-) (from port 2 to port 1) wave propagation directions. Consequently, the difference in phase shifts of the forward and backward propagation directions, $\Delta \varphi=\varphi_{+}-\varphi_{-}$differs from zero, as seen in Fig. 10c for different values of applied field turning this microstrip into a phase shifter device.
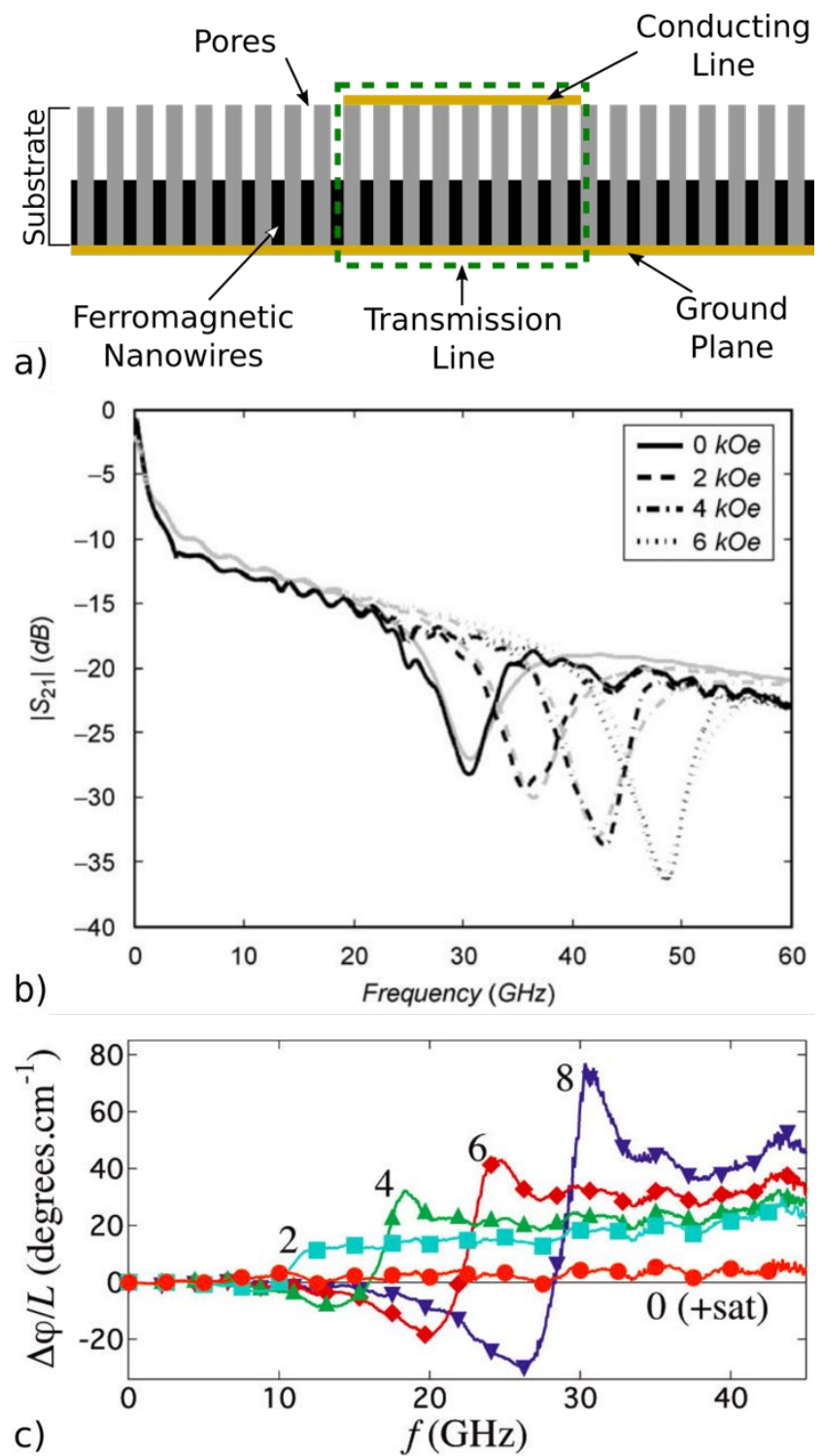

Fig. 10 (a) NWs inside the template as a substrate of a microstrip. (b) Transmission coefficient $\mathrm{S}_{21}$ of CoFe NWs with a DC magnetic field of 2, 4 and $6 \mathrm{kOe}$ applied parallel to the wire axis (Reprinted from [214] Copyright 2008 with permission from Elsevier). (c) Differential phase shift $(\Delta \varphi)$ per total length of the microstrip L at different applied DC magnetic fields from 2 to $8 \mathrm{kOe}$ (Reprinted (adapted) with permission from [218] Copyright 2010 American Chemical Society).
In general, a proper design of the transmission line geometry [219], [220] and substrate's dielectric and magnetic properties [212], [214]-[216] allows the fabrication of circulators [29], [31], filters [221] and phase shifters [32], [218] with a wide frequency range.

\section{SUMMARY, OUTLOOK AND CHALLENGES}

The electrochemical deposition of cylindrical magnetic nanowires into alumina templates is a fabrication method that features precision in terms of NW geometry, control over the NW material, batch fabrication and freedom over various design parameters. As a consequence, it has been utilized in many studies of fundamental properties as well as to implement multiple applications. In this review, we covered the basics of this versatile fabrication method and summarized the various materials and designs that have been realized, leading to different means to tailor the NW properties. Then, we covered the applications of these NWs, starting with the permanent magnetic applications over the low frequency regime, which includes biomedical applications, to high frequency operations as memory elements or electronic components.

The review of these applications revealed that NWs excel in many ways over other implementations, thanks to their unique features. The translation of these research results into commercial products, however, has yet to follow. One obstacle that most of these prospective devices share with many others is that their fabrication process has not been fully integrated into standard Si processing methods, despite efforts in anodizing thin sputtered or evaporated layers of aluminum on prevalent industry substrates (see for example section 7.5 in [48]). Therefore, industrial-scale manufacturers of NWs and membranes with highly reproducible and precise properties are scarce. On the other hand, released NWs are not widely commercially available and are employed in small quantities. Compared to, for example, the high production yields of superparamagnetic nanoparticles, which do not necessarily require a template, the low yield of NWs in large lots limits their widespread application and utilization and imposes a hurdle to enter the research field.

One aspect that needs to be further developed is single crystallinity of NWs. This has not been attained consistently and could allow huge improvements in many applications, especially of NWs used as electrodes, where defects limit charge carrier diffusion lengths. A tighter control over the electroplating conditions could help in this regard too: most research groups work with setups built in-house with variable solution quantities, temperature, stirring and atmospheric conditions. The same issues are faced when fabricating alumina templates using anodization and higher degree of pore-order etc. are desired.

Attempts to use arrays of nanowires as an alternative family of permanent magnets without rare-earth elements have been partly successful in spite of their relevance for energy-related 
applications. Their relevance, arises from the possibility to superimpose the magnetocrystalline or magnetoelastic anisotropies to the geometrical shape anisotropy resulting in stronger total axial magnetic anisotropy, and hence, stronger, tunable magnets. Nevertheless, this total magnetic anisotropy is still low compared to the anisotropy found in rare-earth containing alloys (e.g., FeNdB) which results in their high magnetic coercivity. However, this last one can be enhanced by pinning the domain wall which reverses the NW's magnetization by suitable geometrical (e.g., by sharpening the ends) or compositional (e.g., by growing novel alloys with particular properties as high Curie temperature) designs. In any case, higher packing density of nanowires is needed in order to reach larger magnetization values. This unfortunately introduces significant magnetostatic interactions between the NWs reducing the effective axial anisotropy of the whole nanowire array.

In the area of biomedical applications magnetic NWs are a promising approach toward a practical solution for nano-robots. They offer position control, visualization and multi-modal actuation (vibration, rotation, heating) remotely and enable drug deliver as well as functionalization for specific targeting in a small, customizable package. Combining those capabilities into a single nano platform can result in powerful tools for theranostics or personalized medicine, which has been partly shown in some works already. However, more research is needed to optimize these instruments and better understand the many ways of interaction between the NWs and cells. An inevitable step in the future has to be in-vivo testing of the NWs and long-term studies. A concern that needs to be better addressed is agglomeration of the NWs, due to magnetostatic interactions. Hence, surface coatings need to be further optimized to aid their dispersion and selectivity.

On the other end of the frequency range, 3-dimensional magnetic memories and FMR-based microwave electronics are very promising. They benefit from the template fabrication method, which provides out-of-plane magnetic structures separated by an electrical insulation layer with aspect ratios that cannot be realized by standard Si fabrication technologies. A critical factor for further development of these devices will be the successful integration with standard electronics on-chip. In case of microwave applications of cylindrical magnetic NWs, the field dependence of resonant frequencies could be exploited for sensing applications, which could be especially attractive for wireless sensor networks.

Through the large range of materials and properties that have been developed for cylindrical magnetic NWs, fascinating opportunities have been created, and the number of applications keeps growing. A further impact and technological translation of these NW-based solutions could be obtained by implementing a scaled-up fabrication process that would provide better accessibility to this technology.

\section{ACKNOWLEDGMENTS}

This work has been supported by the Spanish Ministry of Economy, Industry and Competitiveness (MINECO) under project MAT2016-76824-C3-1-R and the Regional Government of Madrid under project S2018/NMT-4321 NANOMAGCOST-CM.

This publication is based upon work supported by the King Abdullah University of Science and Technology (KAUST) Office of Sponsored Research (OSR) under award No. OSR2016-CRG5-2956.

\section{REFERENCES}

[1] A. Fernández-Pacheco, R. Streubel, O. Fruchart, R. Hertel, P. Fischer, and R. P. Cowburn, "Three-dimensional nanomagnetism," Nat. Commun., vol. 8, no. 1, p. 15756, Aug. 2017, doi: $10.1038 /$ ncomms 15756.

[2] A. Mourachkine, O. V. Yazyev, C. Ducati, and J. P. Ansermet, "Template nanowires for spintronics applications: Nanomagnet microwave resonators functioning in zero applied magnetic field," Nano Lett., vol. 8, no. 11, pp. 3683-3687, 2008, doi:

$10.1021 / \mathrm{nl} 801820 \mathrm{~h}$.

[3] X. Kou et al., "Memory effect in magnetic nanowire arrays," Adv. Mater., vol. 23, no. 11, pp. 1393-1397, 2011, doi: 10.1002/adma.201003749.

[4] M. F. Contreras, R. Sougrat, A. Zaher, T. Ravasi, and J. Kosel, "Non-chemotoxic induction of cancer cell death using magnetic nanowires," Int. J. Nanomedicine, vol. 10, pp. 2141-2153, 2015, doi: 10.2147/IJN.S77081.

[5] H. Mohammed, J. A. Moreno, and J. Kosel, "Advanced Fabrication and Characterization of Magnetic Nanowires," in Magnetism and Magnetic Materials, vol. 2, InTech, 2018, pp. 137-164.

[6] Q. Xu, G. Meng, and F. Han, "Porous AAO template-assisted rational synthesis of large-scale 1D hybrid and hierarchically branched nanoarchitectures," Prog. Mater. Sci., vol. 95, pp. 243285, 2018, doi: 10.1016/j.pmatsci.2018.02.004.

[7] M. Staňo and O. Fruchart, "Magnetic Nanowires and Nanotubes," Handb. Magn. Mater., vol. 27, no. 1999, pp. 155-267, 2018, doi: 10.1016/bs.hmm.2018.08.002.

[8] L. Piraux, "Magnetic nanowires," Appl. Sci., vol. 10, no. 5, 2020, doi: 10.3390/app10051832.

[9] M. Vázquez, Ed., Magnetic Nano- and Microwires, 2nd ed. Elsevier, 2020.

[10] M. Jaafar et al., "Customized MFM probes based on magnetic nanorods," Nanoscale, 2020, doi: 10.1039/d0nr00322k.

[11] K. Soulantica, T. Blon, B. Chaudret, L.-M. Lacroix, G. Viau, and F. Ott, From soft chemistry to $2 \mathrm{D}$ and $3 D$ nanowire arrays with hard magnetic properties and permanent magnet applications. 2020.

[12] Y. P. Ivanov et al., "Design of intense nanoscale stray fields and gradients at magnetic nanorod interfaces," ACS Appl. Mater. Interfaces, vol. 11, p. acsami.8b19873, 2019, doi: 10.1021/acsami.8b19873.

[13] A. Alfadhel, B. Li, A. Zaher, O. Yassine, and J. Kosel, “A magnetic nanocomposite for biomimetic flow sensing," Lab Chip, vol. 14, no. 22, pp. 4362-4369, 2014, doi: 10.1039/c4lc00821a.

[14] A. Alfadhel and J. Kosel, "Magnetic Nanocomposite Cilia Tactile Sensor," Adv. Mater., vol. 27, no. 47, pp. 7888-7892, 2015, doi: 10.1002/adma.201504015.

[15] G. Yang et al., "Magnetic nanowire based high resolution magnetic force microscope probes," Appl. Phys. Lett., vol. 87, no. 12, pp. 1-3, 2005, doi: 10.1063/1.2043237.

[16] M. Alnassar, A. Alfadhel, Y. P. Ivanov, and J. Kosel, "Magnetoelectric polymer nanocomposite for flexible electronics," J. Appl. Phys., vol. 117, no. 17, 2015, doi: 10.1063/1.4913943.

[17] M. A. Khan, A. Alfadhel, and J. Kosel, "Magnetic Nanocomposite Cilia Energy Harvester," IEEE Trans. Magn., vol. 52, no. 7, pp. 14, 2016, doi: 10.1109/TMAG.2016.2527733.

[18] F. Mushtaq et al., "Highly Efficient Coaxial TiO 2 -PtPd Tubular Nanomachines for Photocatalytic Water Purification with Multiple Locomotion Strategies," Adv. Funct. Mater., vol. 26, no. 38, pp. 
6995-7002, 2016, doi: 10.1002/adfm.201602315.

[19] B. Jang et al., "Programmable Locomotion Mechanisms of Nanowires with Semihard Magnetic Properties Near a Surface Boundary," ACS Appl. Mater. Interfaces, vol. 11, no. 3, pp. 3214 3223, 2019, doi: 10.1021/acsami.8b16907.

[20] J. Wang, "Barcoded metal nanowires," J. Mater. Chem., vol. 18, no. 34, pp. 4017-4020, 2008, doi: 10.1039/b803807d.

[21] M. Tanase, E. J. Felton, D. S. Gray, A. Hultgren, C. S. Chen, and D. H. Reich, "Assembly of multicellular constructs and microarrays of cells using magnetic nanowires," Lab Chip, vol. 5, no. 6, pp. 598605, 2005, doi: 10.1039/b500243e.

[22] J. Carrey, B. Mehdaoui, and M. Respaud, "Simple models for dynamic hysteresis loop calculations of magnetic single-domain nanoparticles: Application to magnetic hyperthermia optimization," J. Appl. Phys., vol. 109, no. 8, 2011, doi: 10.1063/1.3551582.

[23] N. J. Hogan, A. S. Urban, C. Ayala-Orozco, A. Pimpinelli, P. Nordlander, and N. J. Halas, "Nanoparticles heat through light localization," Nano Lett., vol. 14, no. 8, pp. 4640-4645, 2014, doi: 10.1021/n15016975.

[24] K. M. Krishnan, "Biomedical Nanomagnetics: A Spin Through Possibilities in Imaging, Diagnostics, and Therapy," IEEE Trans. Magn., vol. 46, no. 7, pp. 2523-2558, Jul. 2010, doi: 10.1109/TMAG.2010.2046907.

[25] K. Žužek Rožman, 20 - Functionalization of magnetic nanowires for biomedical applications. 2015.

[26] Y. P. Ivanov et al., "Tunable magnetic nanowires for biomedical and harsh environment applications," Sci. Rep., vol. 6, no. March, p. 24189, 2016, doi: 10.1038/srep24189.

[27] D. Shore et al., "Electrodeposited Fe and Fe-Au nanowires as MRI contrast agents," Chem. Commun., vol. 52, no. 85, pp. 12634 12637, 2016, doi: 10.1039/c6cc06991f.

[28] M. Y. Liao et al., "Synthesis of magnetic hollow nanotubes based on the kirkendall effect for MR contrast agent and colorimetric hydrogen peroxide sensor," J. Mater. Chem., vol. 21, no. 22, pp. 7974-7981, 2011, doi: 10.1039/c1jm10429b.

[29] M. Darques, J. De La Torre Medina, L. Piraux, L. Cagnon, and I. Huynen, "Microwave circulator based on ferromagnetic nanowires in an alumina template," Nanotechnology, vol. 21, no. 14, 2010, doi: 10.1088/0957-4484/21/14/145208.

[30] N. Parsa, M. R. Gasper, V. S. Praneeth Karempudi, and R. C. Toonen, "Magnetic field dependence of non-reciprocal propagation of millimeter-waves through arrays of ferromagnetic nanowires," 2018 IEEE 13th Nanotechnol. Mater. Devices Conf. NMDC 2018, pp. 1-4, 2019, doi: 10.1109/NMDC.2018.8605890.

[31] W. Zhou, J. Um, B. Stadler, and R. Franklin, "Design of self-biased coplanar circulator with ferromagnetic nanowires," IEEE Radio Wirel. Symp. RWS, vol. 2018-Janua, pp. 240-242, 2018, doi: 10.1109/RWS.2018.8304998.

[32] M. Sharma, B. K. Kuanr, M. Sharma, and A. Basu, "New opportunities in microwave electronics with ferromagnetic nanowires," J. Appl. Phys., vol. 115, no. 17, pp. 1-4, 2014, doi: 10.1063/1.4866235.

[33] L. Piraux et al., "Giant magnetoresistance in magnetic multilayered nanowires," Appl. Phys. Lett., vol. 65, no. 19, pp. 2484-2486, 1994, doi: 10.1063/1.112672.

[34] A. Fert and L. Piraux, "Magnetic nanowires," J. Magn. Magn. Mater., vol. 200, no. 1-3, pp. 338-358, Oct. 1999, doi: 10.1016/S0304-8853(99)00375-3.

[35] a. Blondel, J. P. Meier, B. Doudin, and J. P. Ansermet, "Giant magnetoresistance of nanowires of multilayers," Appl. Phys. Lett., vol. 65 , no. 23 , pp. 3019-3021, 1994, doi: 10.1063/1.112495. H. Mohammed, E. V. Vidal, Y. P. Ivanov, and J. Kosel, "Magnetotransport Measurements of Domain Wall Propagation in Individual Multisegmented Cylindrical Nanowires," IEEE Trans. Magn., vol. 52, no. 7, 2016, doi: 10.1109/TMAG.2016.2536644.

[37] C. Bran et al., "Magnetization Ratchet in Cylindrical Nanowires," ACS Nano, vol. 12, no. 6, pp. 5932-5939, Jun. 2018, doi: 10.1021/acsnano.8b02153.

[38] E. Berganza, C. Bran, M. Jaafar, M. Vázquez, and A. Asenjo, "Domain wall pinning in $\mathrm{FeCoCu}$ bamboo-like nanowires," Sci. Rep., vol. 6, no. 1, p. 29702, 2016, doi: 10.1038/srep29702.

[39] L. Piraux et al., "Template-grown $\mathrm{NiFe} / \mathrm{Cu} / \mathrm{NiFe}$ nanowires for spin transfer devices," Nano Lett., vol. 7, no. 9, pp. 2563-2567, 2007, doi: 10.1021/n1070263s.

[40] M. M. Maqableh et al., "Low-resistivity $10 \mathrm{~nm}$ diameter magnetic sensors," Nano Lett., vol. 12, no. 8, pp. 4102-4109, 2012, doi 10.1021/nl301610z.

[41] M. Schöbitz et al., "Fast Domain Wall Motion Governed by Topology and Ersted Fields in Cylindrical Magnetic Nanowires," Phys. Rev. Lett., vol. 123, no. 21, pp. 1-6, 2019, doi: 10.1103/PhysRevLett.123.217201.

[42] Y. P. Ivanov, A. Chuvilin, S. Lopatin, and J. Kosel, "Modulated Magnetic Nanowires for Controlling Domain Wall Motion: Toward 3D Magnetic Memories," ACS Nano, vol. 10, no. 5, pp. 5326-5332, 2016, doi: 10.1021/acsnano.6b01337.

[43] H. Mohammed et al., "Current Controlled Magnetization Switching in Cylindrical Nanowires for High-Density 3D Memory Applications," pp. 1-13, 2018, [Online]. Available: http://arxiv.org/abs/1804.06616.

[44] Y. P. Ivanov, A. Chuvilin, S. Lopatin, H. Mohammed, and J. Kosel, "Direct Observation of Current-Induced Motion of a 3D Vortex Domain Wall in Cylindrical Nanowires," ACS Appl. Mater. Interfaces, vol. 9, no. 20, pp. 16741-16744, May 2017, doi: 10.1021/acsami.7b03404.

[45] A. J. Grutter et al., "Complex Three-Dimensional Magnetic Ordering in Segmented Nanowire Arrays," ACS Nano, vol. 11, no. 8, pp. 8311-8319, 2017, doi: 10.1021/acsnano.7b03488.

[46] G. D. Sulka, Highly Ordered Anodic Porous Alumina Formation by Self-Organized Anodizing. 2008.

[47] G. D. Sulka, A. Brzózka, L. Zaraska, E. Wierzbicka, and A. Brudzisz, "AAO Templates with Different Patterns and Channel Shapes," in Submicron Porous Materials, Cham: Springer International Publishing, 2017, pp. 107-156.

[48] W. Lee and S.-J. Park, "Porous Anodic Aluminum Oxide: Anodization and Templated Synthesis of Functional Nanostructures," Chem. Rev., vol. 114, no. 15, pp. 7487-7556, 2014, doi: $10.1021 / \mathrm{cr} 500002 \mathrm{z}$

[49] L. G. Vivas, J. Escrig, D. G. Trabada, G. A. Badini-Confalonieri, and M. V??zquez, "Magnetic anisotropy in ordered textured Co nanowires," Appl. Phys. Lett., vol. 100, no. 25, pp. 1-4, 2012, doi: 10.1063/1.4729782.

[50] S. Karim et al., "Synthesis of gold nanowires with controlled crystallographic characteristics," Appl. Phys. A Mater. Sci. Process., vol. 84, no. 4, pp. 403-407, 2006, doi: 10.1007/s00339-006-3645-6.

[51] V. P. Parkhutik and V. I. Shershulsky, "Theoretical modelling of porous oxide growth on aluminium," J. Phys. D. Appl. Phys., vol. 25, no. 8, pp. 1258-1263, 1992, doi: 10.1088/0022-3727/25/8/017.

[52] V. V Yuzhakov, H. C. Chang, and A. E. Miller, "Pattern formation during electropolishing," Phys. Rev. B, vol. 56, no. 19, pp. 1260812624, 1997, doi: 10.1103/PhysRevB.56.12608.

[53] A. Rauf, M. Mehmood, M. Asim Rasheed, and M. Aslam, "The effects of electropolishing on the nanochannel ordering of the porous anodic alumina prepared in oxalic acid," J. Solid State Electrochem., vol. 13, no. 2, pp. 321-332, 2009, doi: 10.1007/s10008-008-0550-2.

[54] H. Masuda and K. Fukuda, "Ordered Metal Nanohole Arrays Made by a Two-Step Replication of Honeycomb Structures of Anodic Alumina," Science (80-. )., vol. 268, no. 5216, pp. 1466-1468, Jun. 1995, doi: 10.1126/science.268.5216.1466.

[55] H. Masuda, K. Kanezawa, and K. Nishio, "Fabrication of Ideally Ordered Nanohole Arrays in Anodic Porous Alumina Based on Nanoindentation Using Scanning Probe Microscope," Chem. Lett., vol. 31, no. 12, pp. 1218-1219, 2002, doi: 10.1246/cl.2002.1218. C. Y. Liu, A. Datta, and Y. L. Wang, "Ordered anodic alumina nanochannels on focused-ion-beam-prepatterned aluminum surfaces," Appl. Phys. Lett., vol. 78, no. 1, pp. 120-122, 2001, doi: $10.1063 / 1.1335543$.

[57] J. Choi, K. Nielsch, M. Reiche, R. B. Wehrspohn, and U. Gösele, "Fabrication of monodomain alumina pore arrays with an interpore distance smaller than the lattice constant of the imprint stamp," $J$. Vac. Sci. Technol. B Microelectron. Nanom. Struct., vol. 21, no. 2, p. 763, 2003, doi: 10.1116/1.1556397.

[58] R. Ji, W. Lee, R. Scholz, U. Gösele, and K. Nielsch, “Templated fabrication of nanowire and nanoring arrays based on interference lithography and electrochemical deposition," Adv. Mater., vol. 18, no. 19 , pp. 2593-2596, 2006, doi: 10.1002/adma.200601136.

[59] W. Lee, R. Ji, C. A. Ross, U. Gösele, and K. Nielsch, "Wafer-Scale Ni Imprint Stamps for Porous Alumina Membranes Based on Interference Lithography," Small, vol. 2, no. 8-9, pp. 978-982, 2006, doi: 10.1002/smll.200600100. 
[60] W. Lee, R. Ji, U. Gösele, and K. Nielsch, "Fast fabrication of longrange ordered porous alumina membranes by hard anodization," Nat. Mater., vol. 5, no. 9, pp. 741-747, 2006, doi: 10.1038/nmat1717.

[61] S. Ono, M. Saito, and H. Asoh, "Self-Ordering of Anodic Porous Alumina Induced by Local Current Concentration: Burning," Electrochem. Solid-State Lett., vol. 7, no. 7, p. B21, 2004, doi: $10.1149 / 1.1738553$.

[62] W. Lee, K. Schwirn, M. Steinhart, E. Pippel, R. Scholz, and U. Gösele, "Structural engineering of nanoporous anodic aluminium oxide by pulse anodization of aluminium," Nat. Nanotechnol., vol. 3, no. 4, pp. 234-239, Apr. 2008, doi: 10.1038/nnano.2008.54.

[63] D. Losic, M. Lillo, and D. Losic, "Porous Alumina with Shaped Pore Geometries and Complex Pore Architectures Fabricated by Cyclic Anodization,” Small, vol. 5, no. 12, pp. 1392-1397, Jun. 2009, doi: 10.1002/smll.200801645.

[64] M. H.-V. and K. N. I Minguez-Bacho, S Rodriguez-López, M Vázquez, "Electrochemical synthesis and magnetic characterization of periodically modulated Co nanowires," Nanotechnology, vol. 25 , no. 14, p. 145301, 2014, doi: 10.1088/0957-4484/25/14/145301.

[65] B. Abad, J. Maiz, and M. Martin-Gonzalez, "Rules to Determine Thermal Conductivity and Density of Anodic Aluminum Oxide (AAO) Membranes," J. Phys. Chem. C, vol. 120, no. 10, pp. 53615370, 2016, doi: 10.1021/acs.jpcc.6b00643.

[66] A. P. Li, F. Müller, A. Birner, K. Nielsch, and U. Gösele, "Polycrystalline nanopore arrays with hexagonal ordering on aluminum," J. Vac. Sci. Technol. A Vacuum, Surfaces, Film., vol. 17, no. 4, p. 1428, 1999, doi: 10.1116/1.581832.

[67] A. P. Li, F. Müller, A. Birner, K. Nielsch, and U. Gösele, "Hexagonal pore arrays with a 50-420 nm interpore distance formed by self-organization in anodic alumina," J. Appl. Phys., vol. 84, no. 11, pp. 6023-6026, Dec. 1998, doi: 10.1063/1.368911.

[68] S. Z. Chu, K. Wada, S. Inoue, M. Isogai, and A. Yasumori, "Fabrication of ideally ordered nanoporous alumina films and integrated alumina nanotubule arrays by high-field anodization," Adv. Mater., vol. 17, no. 17, pp. 2115-2119, 2005, doi: 10.1002/adma.200500401

[69] S. Z. Chu, K. Wada, S. Inoue, M. Isogai, Y. Katsuta, and A. Yasumori, "Large-Scale Fabrication of Ordered Nanoporous Alumina Films with Arbitrary Pore Intervals by Critical-Potential Anodization," J. Electrochem. Soc., vol. 153, no. 9, p. B384, 2006, doi: $10.1149 / 1.2218822$

[70] L. Zaraska, W. J. Stępniowski, G. D. Sulka, E. Ciepiela, and M. Jaskuła, "Analysis of nanopore arrangement and structural features of anodic alumina layers formed by two-step anodizing in oxalic acid using the dedicated executable software," Appl. Phys. A Mater. Sci. Process., vol. 114, no. 2, pp. 571-577, 2014, doi: 10.1007/s00339-013-7618-2.

[71] O. Jessensky, F. Müller, and U. Gösele, "Self-organized formation of hexagonal pore arrays in anodic alumina," Appl. Phys. Lett., vol. 72, no. 10, pp. 1173-1175, 1998, doi: 10.1063/1.121004.

[72] C. Sun, J. Luo, L. Wu, and J. Zhang, "Self-ordered anodic alumina with continuously tunable pore intervals from 410 to $530 \mathrm{~nm}$," ACS Appl. Mater. Interfaces, vol. 2, no. 5, pp. 1299-1302, 2010, doi: 10.1021/am1001713.

[73] V. Mizeikis, I. Mikulskas, R. Tomašiunas, S. Juodkazis, S. Matsuo, and H. Misawa, "Optical characteristics of two-dimensional photonic crystals in anodic aluminum oxide films," Japanese J. Appl. Physics, Part 1 Regul. Pap. Short Notes Rev. Pap., vol. 43, no. 6 A, pp. 3643-3647, 2004, doi: 10.1143/JJAP.43.3643.

[74] A. Cuevas, M. Martínez de Yuso, V. Vega, A. González, V. Prida, and J. Benavente, "Influence of ALD Coating Layers on the Optical Properties of Nanoporous Alumina-Based Structures," Coatings, vol. 9, no. 1, p. 43, 2019, doi: 10.3390/coatings9010043.

[75] L. Zhou et al., "Self-assembly of highly efficient, broadband plasmonic absorbers for solar steam generation," Sci. Adv., vol. 2, no. 4, pp. e1501227-e1501227, 2016, doi: 10.1126/sciadv.1501227. G. Marinaro et al., "Plasmonic nanowires for wide wavelength range molecular sensing," Materials (Basel)., vol. 11, no. 5, pp. 113, 2018, doi: 10.3390/ma11050827.

[77] M. Schlesinger and M. Paunovic, Modern Electroplating: Fifth Edition. 2011.

[78] ASTM B689-97(2018), "Standard Specification for Electroplated Engineering Nickel Coatings," West Conshohocken, PA, 2018. doi: 10.1520/B0689-97R18.
[79] G. A. Di Bari, "Electrodeposition of Nickel," in Modern Electroplating, no. February, E. Milan, Ed. Hoboken, NJ, USA: John Wiley \& Sons, Inc., 2011, pp. 79-114.

[80] A. Saraby-Reintjes and M. Fleischmann, "Kinetics of electrodeposition of nickel from watts baths," Electrochim. Acta, vol. 29 , no. 4, pp. 557-566, 1984, doi: 10.1016/00134686(84)87109-1.

[81] J. W. Dini and D. D. Snyder, "Electrodeposition of Copper," in Modern Electroplating, vol. 47, Hoboken, NJ, USA: John Wiley \& Sons, Inc., 2011, pp. 33-78.

[82] P. A. Kohl, "Electrodeposition of Gold," in Modern Electroplating, no. February, J. W. \& Sons, Ed. Hoboken, NJ, USA: John Wiley \& Sons, Inc., 2011, pp. 115-130.

[83] M. Izaki, "Electrodeposition of Iron and Iron Alloys," Mod. Electroplat., pp. 309-326, 2011, doi:

10.1002/9780470602638.ch11.

[84] C. Q. Cui, "Electrodeposition of Cobalt from Aqueous Chloride Solutions," J. Electrochem. Soc., vol. 137, no. 11, p. 3418, 1990, doi: 10.1149/1.2086232.

[85] J. S. Santos, R. Matos, F. Trivinho-Strixino, and E. C. Pereira, "Effect of temperature on Co electrodeposition in the presence of boric acid," Electrochim. Acta, vol. 53, no. 2, pp. 644-649, 2007, doi: $10.1016 /$ j.electacta.2007.07.025.

[86] R. Saeki and T. Ohgai, "Effect of Growth Rate on the Crystal Orientation and Magnetization Performance of Cobalt Nanocrystal Arrays Electrodeposited from Aqueous Solution," Nanomaterials, vol. 8, no. 8, p. 566, 2018, doi: 10.3390/nano8080566.

[87] J. Yahalom, D. F. Tessier, R. S. Timsit, A. M. Rosenfeld, D. F. Mitchell, and P. T. Robinson, "Structure of composition-modulated $\mathrm{Cu} / \mathrm{Ni}$ thin films prepared by electrodeposition," Journal of Materials Research, vol. 4, no. 04. pp. 755-758, 1989, doi: 10.1557/JMR.1989.0755.

[88] Y. P. Ivanov, D. G. Trabada, A. Chuvilin, J. Kosel, O. ChubykaloFesenko, and M. Vázquez, "Crystallographically driven magnetic behaviour of arrays of monocrystalline Co nanowires.," Nanotechnology, vol. 25, no. 47, p. 475702, 2014, doi: 10.1088/0957-4484/25/47/475702.

[89] A. K. Srivastav and R. Shekhar, "Crystal anisotropy induced temperature dependent magnetization in cobalt nanowires electrodeposited within alumina template," J. Magn. Magn. Mater., vol. 349, pp. 21-26, 2014, doi: 10.1016/j.jmmm.2013.08.041.

[90] M. Méndez et al., "Ni-Co Alloy and Multisegmented Ni/Co Nanowire Arrays Modulated in Composition: Structural Characterization and Magnetic Properties," Crystals, vol. 7, no. 3, p. 66, 2017, doi: 10.3390/cryst7030066.

[91] Ó. Iglesias-Freire et al., "Spin configuration in isolated $\mathrm{FeCoCu}$ nanowires modulated in diameter," Nanotechnology, vol. 26, no. 39, p. 395702, 2015, doi: 10.1088/0957-4484/26/39/395702.

[92] F. Tejo, N. Vidal-Silva, A. P. Espejo, and J. Escrig, "Angular dependence of the magnetic properties of cylindrical diameter modulated Ni80Fe20 nanowires," J. Appl. Phys., vol. 115, no. 17, 2014, doi: 10.1063/1.4865777.

[93] L. A. Rodríguez et al., "Quantitative Nanoscale Magnetic Study of Isolated Diameter-Modulated FeCoCu Nanowires," ACS Nano, vol. 10, no. 10, pp. 9669-9678, 2016, doi: 10.1021/acsnano.6b05496.

[94] E. Ortega, S. M. Reddy, I. Betancourt, S. Roughani, B. J. H. Stadler, and A. Ponce, "Magnetic ordering in $45 \mathrm{~nm}$-diameter multisegmented $\mathrm{FeGa} / \mathrm{Cu}$ nanowires: Single nanowires and arrays," J. Mater. Chem. C, vol. 5, no. 30, pp. 7546-7552, 2017, doi: $10.1039 / \mathrm{c} 7 \mathrm{tc} 02314 \mathrm{f}$.

[95] Y. P. Ivanov, L. G. Vivas, a. Asenjo, a. Chuvilin, O. Chubykalofesenko, and M. Vázquez, "Magnetic structure of a single-crystal hcp electrodeposited cobalt nanowire," EPL (Europhysics Lett., vol. 102, no. 1, p. 17009, 2013, doi: 10.1209/0295-5075/102/17009.

[96] Y. P. Ivanov, A. Chuvilin, L. G. Vivas, J. Kosel, O. ChubykaloFesenko, and M. Vázquez, "Single crystalline cylindrical nanowires - toward dense 3D arrays of magnetic vortices," Sci. Rep., vol. 6, no. March, p. 23844, 2016, doi: 10.1038/srep23844.

[97] M. Paunovic, M. Schlesinger, and D. D. Snyder, "Fundamental Considerations," in Modern Electroplating, M. Schlesinger and M. Paunovic, Eds. Hoboken, NJ, USA: John Wiley \& Sons, Inc., 2011, pp. 1-32.

[98] K. Nielsch, F. Müller, A. P. Li, and U. Gösele, "Uniform nickel deposition into ordered alumina pores by pulsed electrodeposition," Adv. Mater., vol. 12, no. 8, pp. 582-586, 2000, doi: 
10.1002/(SICI)1521-4095(200004)12:8<582::AIDADMA582>3.0.CO;2-3.

[99] Y. Hu, A. Kleiman-Shwarsctein, A. J. Forman, D. Hazen, J. Park, and E. W. McFarland, "Pt-Doped $\alpha$-Fe 2 O 3 Thin Films Active for Photoelectrochemical Water Splitting," Chem. Mater., vol. 20, no. 12, pp. 3803-3805, Jun. 2008, doi: 10.1021/cm800144q.

[100] E. Vilanova Vidal, Y. P. Ivanov, H. Mohammed, and J. Kosel, “A detailed study of magnetization reversal in individual $\mathrm{Ni}$ nanowires," Appl. Phys. Lett., vol. 106, no. 3, p. 032403, 2015, doi: 10.1063/1.4906108.

[101] N. Yasui, A. Imada, and T. Den, "Electrodeposition of (001) oriented CoPtL10columns into anodic alumina films," Appl. Phys. Lett., vol. 83, no. 16, pp. 3347-3349, 2003, doi: 10.1063/1.1622787.

[102] A. I. Martínez-Banderas et al., "Functionalized magnetic nanowires for chemical and magneto-mechanical induction of cancer cell death,"Sci. Rep., vol. 6, no. October, pp. 1-11, 2016, doi: 10.1038/srep35786.

[103] M. S. Salem, P. Sergelius, R. M. Corona, J. Escrig, D. Görlitz, and K. Nielsch, "Magnetic properties of cylindrical diameter modulated Ni80Fe20 nanowires: interaction and coercive fields," Nanoscale, vol. 5, no. 9, p. 3941, 2013, doi: 10.1039/c3nr00633f.

[104] F. Z. Wang et al., "Doubling of the magnetic energy product in ferromagnetic nanowires at ambient temperature by capping their tips with an antiferromagnet," Nanotechnology, vol. 28, no. 29, 2017, doi: 10.1088/1361-6528/aa77b7.

[105] S. Samanifar, M. Almasi Kashi, A. Ramazani, and M. Alikhani, "Reversal modes in FeCoNi nanowire arrays: Correlation between magnetostatic interactions and nanowires length," J. Magn. Magn. Mater., vol. 378, pp. 73-83, 2015, doi: 10.1016/j.jmmm.2014.10.155.

[106] E. M. Palmero, C. Bran, R. P. Del Real, and M. Vázquez, "Vortex domain wall propagation in periodically modulated diameter $\mathrm{FeCoCu}$ nanowire as determined by the magneto-optical Kerr effect," Nanotechnology, vol. 26, no. 46, 2015, doi: 10.1088/09574484/26/46/461001.

[107] Y. H. Huang, H. Okumura, G. C. Hadjipanayis, and D. Weller, "CoPt and FePt nanowires by electrodeposition," J. Appl. Phys., vol. 91, no. 10 , p. 6869,2002 , doi: $10.1063 / 1.1447524$.

[108] E. M. Palmero et al., "Stepwise magnetization reversal of geometrically tuned in diameter $\mathrm{Ni}$ and $\mathrm{FeCo}$ bi-segmented nanowire arrays," Nano Res., vol. 12, no. 1, pp. 1-7, Apr. 2019, doi: 10.1007/s12274-019-2385-9.

[109] M. Chen, P. C. Searson, and C. L. Chien, "Micromagnetic behavior of electrodeposited Ni/Cu multilayer nanowires," J. Appl. Phys., vol. 93, no. 103 , pp. 8253-8255, 2003, doi: 10.1063/1.1556136.

[110] H. P. Liang, Y. G. Guo, J. S. Hu, C. F. Zhu, L. J. Wan, and C. L. Bai, "Ni-Pt multilayered nanowire arrays with enhanced coercivity and high remanence ratio," Inorg. Chem., vol. 44, no. 9, pp. 30133015, 2005, doi: 10.1021/ic0500917.

[111] P. Sergelius et al., "Intra-wire coupling in segmented $\mathrm{Ni} / \mathrm{Cu}$ nanowires deposited by electrodeposition," Nanotechnology, vol. 28, no. 6, p. 065709, Feb. 2017, doi: 10.1088/1361-6528/aa5118.

[112] B. C. Park and Y. K. Kim, "Synthesis, microstructure, and physical properties of metallic barcode nanowires," Met. Mater. Int., vol. 23, no. 3, pp. 1-13, 2017, doi: 10.1007/s12540-017-7071-4.

[113] M. S. Salem et al., "Composition and diameter modulation of magnetic nanowire arrays fabricated by a novel approach," Nanotechnology, vol. 29, no. 6, p. 065602, Feb. 2018, doi: 10.1088/1361-6528/aaa095.

[114] A. Encinas-Oropesa, M. Demand, L. Piraux, I. Huynen, and U. Ebels, "Dipolar interactions in arrays of nickel nanowires studied by ferromagnetic resonance," Phys. Rev. B - Condens. Matter Mater. Phys., vol. 63, no. 10, pp. 1044151-1044156, 2001, doi: 10.1103/PhysRevB.63.104415.

[115] E. M. Palmero et al., "Enhanced magnetocrystalline anisotropy of Fe30Co70 nanowires by $\mathrm{Cu}$ additives and annealing," Nanotechnology, vol. 27, no. 36, 2016, doi: 10.1088/09574484/27/36/365704.

[116] J. L. Fu, Z. J. Yan, Y. Xu, X. L. Fan, and D. S. Xue, "Effects of annealing temperature on structure and magnetic properties of amorphous Fe61Co27P12 nanowire arrays," J. Phys. Chem. Solids, vol. 68 , no. 12 , pp. 2221-2226, 2007, doi:

10.1016/j.jpcs.2007.06.023.

[117] Z. Liu, W. L. Li, P. P. Jin, and W. D. Fei, "Magnetic anisotropy transformation of $\mathrm{Co}-\mathrm{Fe}-\mathrm{B}$ nanowire arrays synthesized under the magnetic field," J. Magn. Magn. Mater., vol. 345, pp. 96-99, Nov. 2013, doi: 10.1016/j.jmmm.2013.06.043.

[118] C. Bran et al., "Tuning the magnetization reversal process of FeCoCu nanowire arrays by thermal annealing," J. Appl. Phys., vol. 114, no. 4, 2013, doi: 10.1063/1.4816479.

[119] C. Bran, E. M. Palmero, R. P. Del Real, and M. Vazquez, "CoFeCu electroplated nanowire arrays: Role of composition and annealing on structure and magnetic properties," Phys. Status Solidi Appl. Mater. Sci., vol. 211, no. 5, pp. 1076-1082, 2014, doi:

10.1002/pssa.201300766.

[120] S. Liébana Viñas et al., "Magnetic hardening of Fe30Co70nanowires," Nanotechnology, vol. 26, no. 41, 2015, doi: 10.1088/0957-4484/26/41/415704.

[121] A. P. Roberts, C. R. Pike, and K. L. Verosub, "First-order reversal curve diagrams: A new tool for characterizing the magnetic properties of natural samples," J. Geophys. Res. Solid Earth, vol. 105, no. B12, pp. 28461-28475, 2000, doi: 10.1029/2000JB900326.

[122] F. Bron, L.-P. Carignan, D. Mnard, and A. Yelo, "Extracting Individual Properties from Global Behaviour: First-order Reversal Curve Method Applied to Magnetic Nanowire Arrays," in Electrodeposited Nanowires and their Applications, InTech, 2010.

[123] C. I. Dobrotă and A. Stancu, "What does a first-order reversal curve diagram really mean? A study case: Array of ferromagnetic nanowires," J. Appl. Phys., vol. 113, no. 4, 2013, doi: 10.1063/1.4789613.

[124] P. R. Downey, A. B. Flatau, P. D. McGary, and B. J. H. Stadler, "Effect of magnetic field on the mechanical properties of magnetostrictive iron-gallium nanowires," J. Appl. Phys., vol. 103, no. 7, pp. 8-11, 2008, doi: 10.1063/1.2837276.

[125] M. A. Hein et al., "Fabrication of BioInspired Inorganic Nanocilia Sensors," IEEE Trans. Magn., vol. 49, no. 1, pp. 191-196, Jan. 2013, doi: 10.1109/TMAG.2012.2224852.

[126] J. A. Moreno et al., "Growth of Ordered Iron Oxide Nanowires for Photo-electrochemical Water Oxidation," ACS Appl. Energy Mater., vol. 2, no. 12, pp. 8473-8480, Dec. 2019, doi: 10.1021/acsaem.9b01343.

[127] A. Alfadhel and J. Kosel, "Magnetic micropillar sensors for force sensing," SAS 2015 - 2015 IEEE Sensors Appl. Symp. Proc., vol. 4, no. 1, pp. 1-4, 2015, doi: 10.1109/SAS.2015.7133654.

[128] P. D. McGary, L. Tan, J. Zou, B. J. H. Stadler, P. R. Downey, and A. B. Flatau, "Magnetic nanowires for acoustic sensors (invited)," $J$ Appl. Phys., vol. 99, no. 8, 2006, doi: 10.1063/1.2167332.

[129] J. J. Park, K. S. M. Reddy, B. Stadler, and A. Flatau, "Magnetostrictive $\mathrm{Fe}-\mathrm{Ga} / \mathrm{Cu}$ Nanowires Array With GMR Sensor for Sensing Applied Pressure," IEEE Sens. J., vol. 17, no. 7, pp. 2015-2020, 2017, doi: 10.1109/JSEN.2017.2657789.

[130] K. Keshoju and L. Sun, "Mechanical characterization of magnetic nanowire-polydimethylsiloxane composites," J. Appl. Phys., vol. 105, no. 2, 2009, doi: 10.1063/1.3068173.

[131] A. Alfadhel, M. A. Khan, S. Cardoso, D. Leitao, and J. Kosel, "A magnetoresistive tactile sensor for harsh environment applications," Sensors (Switzerland), vol. 16, no. 5, 2016, doi: 10.3390/s16050650.

[132] A. Alfadhel, M. A. Khan, S. Cardoso De Freitas, and J. Kosel, "Magnetic tactile sensor for braille reading," IEEE Sens. J., vol. 16, no. 24, pp. 8700-8705, 2016, doi: 10.1109/JSEN.2016.2558599.

[133] A. Alfadhel, M. A. Khan, S. Cardoso, and J. Kosel, "A single magnetic nanocomposite cilia force sensor," SAS 2016 - Sensors Appl. Symp. Proc., pp. 106-109, 2016, doi:

10.1109/SAS.2016.7479828.

[134] C. Li, Z. Luo, T. Wang, and J. Gong, "Surface, Bulk, and Interface: Rational Design of Hematite Architecture toward Efficient PhotoElectrochemical Water Splitting," Adv. Mater., vol. 1707502, no. 001, pp. 1-23, 2018, doi: 10.1002/adma.201707502.

[135] A. Mao, N. G. Park, G. Y. Han, and J. H. Park, "Controlled growth of vertically oriented hematite/Pt composite nanorod arrays: Use for photoelectrochemical water splitting," Nanotechnology, vol. 22, no. 17, 2011, doi: 10.1088/0957-4484/22/17/175703.

[136] A. Mao, K. Shin, J. K. Kim, D. H. Wang, G. Y. Han, and J. H. Park, "Controlled synthesis of vertically aligned hematite on conducting substrate for photoelectrochemical cells: Nanorods versus nanotubes," ACS Appl. Mater. Interfaces, vol. 3, no. 6, pp. 18521858, 2011, doi: 10.1021/am200407t.

[137] Q. Wei, Y. Fu, G. Zhang, D. Yang, G. Meng, and S. Sun, "Rational design of novel nanostructured arrays based on porous AAO 
templates for electrochemical energy storage and conversion," Nano Energy, vol. 55, no. November 2018, pp. 234-259, 2019, doi: 10.1016/j.nanoen.2018.10.070.

[138] J. P. Heremans, "Review of thermoelectric and galvanomagnetic transport in bismuth nanowires," Int. Conf. Thermoelectr. ICT, Proc., vol. 2003-Janua, pp. 324-329, 2003, doi: 10.1109/ICT.2003.1287515.

[139] A. Isaac Martínez Banderas et al., "Iron-Based Core-Shell Nanowires for Combinatorial Drug Delivery, Photothermal and Magnetic Therapy," ACS Appl. Mater. \&amp; Interfaces, vol. 0, no. ja, 2019, doi: 10.1021/acsami.9b17512.

[140] C. M. Wang et al., "Void formation during early stages of passivation: Initial oxidation of iron nanoparticles at room temperature," J. Appl. Phys., vol. 98, no. 9, 2005, doi: $10.1063 / 1.2130890$.

[141] K. Gandha, R. P. Chaudhary, J. Mohapatra, A. R. Koymen, and J. P. Liu, "Giant exchange bias and its angular dependence in $\mathrm{Co} / \mathrm{CoO}$ core-shell nanowire assemblies," Phys. Lett. Sect. A Gen. At. Solid State Phys., vol. 381, no. 25-26, pp. 2092-2096, 2017, doi: 10.1016/j.physleta.2017.04.025.

[142] Q. Feng, Y. Liu, J. Huang, K. Chen, J. Huang, and K. Xiao, "Uptake, distribution, clearance, and toxicity of iron oxide nanoparticles with different sizes and coatings," Sci. Rep., vol. 8, no. 1, pp. 1-13, 2018, doi: 10.1038/s41598-018-19628-z.

[143] R. A. Revia and M. Zhang, "Magnetite nanoparticles for cancer diagnosis, treatment, and treatment monitoring: recent advances," Mater. Today, vol. 19, no. 3, pp. 157-168, 2016, doi: https://doi.org/10.1016/j.mattod.2015.08.022.

[144] M. P. Raphael, J. A. Christodoulides, S. N. Qadri, B. S. Simpkins, and J. M. Byers, "Magnetic moment degradation of nanowires in biological media: Real-time monitoring with SQUID magnetometry," Nanotechnology, vol. 21, no. 28, 2010, doi: 10.1088/0957-4484/21/28/285101.

[145] D. Bobo, K. J. Robinson, J. Islam, K. J. Thurecht, and S. R. Corrie, "Nanoparticle-Based Medicines: A Review of FDA-Approved Materials and Clinical Trials to Date," Pharm. Res., vol. 33, no. 10, pp. 2373-2387, 2016, doi: 10.1007/s11095-016-1958-5.

[146] G. Luongo et al., "Scalable High-Affinity Stabilization of Magnetic Iron Oxide Nanostructures by a Biocompatible Antifouling Homopolymer," ACS Appl. Mater. Interfaces, vol. 9, no. 46, pp. 40059-40069, Nov. 2017, doi: 10.1021/acsami.7b12290.

[147] W. Zhou et al., "Development of a Biolabeling System Using Ferromagnetic Nanowires," IEEE J. Electromagn. RF Microwaves Med. Biol., vol. 3, no. 2, pp. 1-1, 2018, doi: 10.1109/jerm.2018.2889049.

[148] A. Hultgren, M. Tanase, C. S. Chen, and D. H. Reich, "High-yield cell separations using magnetic nanowires," IEEE Trans. Magn., vol. 40, no. 4 II, pp. 2988-2990, 2004, doi: 10.1109/TMAG.2004.830406.

[149] A. Hultgren et al., "Optimization of yield in magnetic cell separations using nickel nanowires of different lengths," Biotechnol. Prog., vol. 21, no. 2, pp. 509-515, 2005, doi: 10.1021/bp049734w.

[150] A. Prina-Mello, Z. Diao, and J. M. D. Coey, "Internalization of ferromagnetic nanowires by different living cells," $J$. Nanobiotechnology, vol. 4, pp. 1-11, 2006, doi: 10.1186/14773155-4-9.

[151] M. M. Song et al., "Cytotoxicity and cellular uptake of iron nanowires," Biomaterials, vol. 31, no. 7, pp. 1509-1517, 2010, doi: 10.1016/j.biomaterials.2009.11.034.

[152] M. B. Margineanu et al., "Semi-automated quantification of living cells with internalized nanostructures," J. Nanobiotechnology, vol. 14, no. 1, pp. 1-13, 2016, doi: 10.1186/s12951-015-0153-x.

[153] J. E. Perez et al., "Cytotoxicity and intracellular dissolution of nickel nanowires," Nanotoxicology, vol. 10, no. 7, pp. 871-880, 2016, doi: 10.3109/17435390.2015.1132343.

[154] M. Safi et al., "Interactions between magnetic nanowires and living cells: Uptake, toxicity, and degradation," ACS Nano, vol. 5, no. 7, pp. 5354-5364, 2011, doi: 10.1021/nn201121e.

[155] L. Zhang, T. Petit, K. E. Peyer, and B. J. Nelson, "Targeted cargo delivery using a rotating nickel nanowire," Nanomedicine Nanotechnology, Biol. Med., vol. 8, no. 7, pp. 1074-1080, 2012, doi: 10.1016/j.nano.2012.03.002.

[156] L. Zhang et al., "Controlled propulsion and cargo transport of rotating nickel nanowires near a patterned solid surface," $A C S$ Nano, vol. 4, no. 10, pp. 6228-6234, 2010, doi:
$10.1021 / \mathrm{nn} 101861 \mathrm{n}$

[157] W. Gao et al., "Cargo-towing fuel-free magnetic nanoswimmers for targeted drug delivery," Small, vol. 8, no. 3, pp. 460-467, 2012, doi: 10.1002/smll.201101909.

[158] N. A. Alsharif, A. Martiinez-Banderas, J. Merzaban, T. Ravasi, and J. Kosel, "Biofunctionalizing Magnetic Nanowires Toward Targeting and Killing Leukemia Cancer Cells," IEEE Trans. Magn., vol. 55, no. 2, pp. 2-6, 2019, doi: 10.1109/TMAG.2018.2864245.

[159] N. Gao, H. Wang, and E. H. Yang, "An experimental study on ferromagnetic nickel nanowires functionalized with antibodies for cell separation," Nanotechnology, vol. 21, no. 10, 2010, doi: 10.1088/0957-4484/21/10/105107.

[160] O. Yassine et al., "Magnetically Triggered Monodispersed Nanocomposite Fabricated by Microfluidic Approach for Drug Delivery," Int. J. Polym. Sci., vol. 2016, pp. 1-8, 2016, doi: 10.1155/2016/1219469.

[161] O. Yassine et al., "Highly Efficient Thermoresponsive Nanocomposite for Controlled Release Applications," Sci. Rep., vol. 6, pp. 1-7, 2016, doi: 10.1038/srep28539.

[162] P. W. Egolf et al., "Hyperthermia with rotating magnetic nanowires inducing heat into tumor by fluid friction," J. Appl. Phys., vol. 120, no. 6, 2016, doi: 10.1063/1.4960406.

[163] L. Yang, N. Zhao, and D. Liu, "Dynamics of ferromagnetic nanowires in a rotating magnetic field," Adv. Mech. Eng., vol. 7, no. 7, pp. 1-11, 2015, doi: 10.1177/1687814015589686.

[164] D. S. Choi et al., "Hyperthermia with Magnetic Nanowires for Inactivating Living Cells," J. Nanosci. Nanotechnol., vol. 8, no. 5, pp. 2323-2327, May 2008, doi: 10.1166/jnn.2008.273.

[165] G. Grile, "The Effects of Heat and Radiation on Cancers Implanted on the Feet of Mice," Cancer Res., vol. 23, no. 3, pp. 372-380, 1963, [Online]. Available: http://cancerres.aacrjournals.org/content/23/3/372.abstract.

[166] R. R. Wildeboer, P. Southern, and Q. A. Pankhurst, "On the reliable measurement of specific absorption rates and intrinsic loss parameters in magnetic hyperthermia materials," J. Phys. D. Appl. Phys., vol. 47, no. 49, 2014, doi: 10.1088/0022-3727/47/49/495003.

[167] J. Alonso et al., "FeCo nanowires with enhanced heating powers and controllable dimensions for magnetic hyperthermia," J. Appl. Phys., vol. 117, no. 17, 2015, doi: 10.1063/1.4908300.

[168] D. Shore et al., "Nanowarming using Au-tipped Co $35 \mathrm{Fe} 65$ ferromagnetic nanowires ," Nanoscale, vol. 11, no. 31, 2019, doi: $10.1039 / \mathrm{c} 9 \mathrm{nr} 01182 \mathrm{j}$.

[169] M. Castillo, R. Ebensperger, D. Wirtz, M. Walczak, D. E. Hurtado, and A. Celedon, "Local mechanical response of cells to the controlled rotation of magnetic nanorods," J. Biomed. Mater. Res. Part B Appl. Biomater., vol. 102, no. 8, pp. 1779-1785, 2014, doi: 10.1002/jbm.b.33167.

[170] A. O. Fung, V. Kapadia, E. Pierstorff, D. Ho, and Y. Chen, "Induction of cell death by magnetic actuation of nickel nanowires internalized by fibroblasts," J. Phys. Chem. C, vol. 112, no. 39, pp. 15085-15088, 2008, doi: 10.1021/jp806187r.

[171] D. S. Choi, X. Hopkins, R. Kringel, J. Park, I. T. Jeon, and Y. Keun Kim, "Magnetically driven spinning nanowires as effective materials for eradicating living cells," J. Appl. Phys., vol. 111, no. 7, 2012, doi: 10.1063/1.3678437.

[172] A. J. Aaron, A. Bumb, and M. W. Brechbiel, "Macromolecules, dendrimers, and nanomaterials in magnetic resonance imaging: The interplay between size, function, and pharmacokinetics," Chem. Rev., vol. 110, no. 5, pp. 2921-2959, 2010, doi: 10.1021/cr900232t.

[173] Y. D. Xiao, R. Paudel, J. Liu, C. Ma, Z. S. Zhang, and S. K. Zhou, "MRI contrast agents: Classification and application (Review)," Int. J. Mol. Med., vol. 38, no. 5, pp. 1319-1326, 2016, doi: 10.3892/ijmm.2016.2744.

[174] U. I. Tromsdorf et al., "Size and surface effects on the MRI relaxivity of manganese ferrite nanoparticle contrast agents," Nano Lett., vol. 7, no. 8, pp. 2422-2427, 2007, doi: 10.1021/n1071099b.

[175] E. D. Smolensky et al., "Scaling laws at the nanosize: The effect of particle size and shape on the magnetism and relaxivity of iron oxide nanoparticle contrast agents," J. Mater. Chem. B, vol. 1, no. 22, pp. 2818-2828, 2013, doi: 10.1039/c3tb00369h.

[176] A. I. Martínez-Banderas et al., "Magnetic core-shell nanowires as MRI contrast agents for cell tracking," J. Nanobiotechnology, vol. 18, no. 1, pp. 1-12, 2020, doi: 10.1186/s12951-020-00597-3.

[177] J. E. Perez, T. Ravasi, and J. Kosel, "Corrigendum: Mesenchymal stem cells cultured on magnetic nanowire substrates (2017 
Nanotechnology 28 055703)," Nanotechnology, vol. 28, no. 50, p. 509501, Dec. 2017, doi: 10.1088/1361-6528/aa966b.

[178] M. Bañobre-López, C. Bran, C. Rodríguez-Abreu, J. Gallo, M. Vázquez, and J. Rivas, "A colloidally stable water dispersion of $\mathrm{Ni}$ nanowires as an efficient: T 2-MRI contrast agent," J. Mater. Chem. $B$, vol. 5, no. 18 , pp. 3338-3347, 2017, doi: 10.1039/c7tb00574a.

[179] J. Mohapatra, A. Mitra, H. Tyagi, D. Bahadur, and M. Aslam, "Iron oxide nanorods as high-performance magnetic resonance imaging contrast agents," Nanoscale, vol. 7, no. 20, pp. 9174-9184, 2015, doi: $10.1039 / \mathrm{c} 5 \mathrm{nr} 00055 \mathrm{f}$.

[180] Horwitz, E. M. et al., "Transplantability and therapeutic effects of bone marrow-derived mesenchymal cells in children with osteogenesis imperfecta," Nat. Med., vol. 5, no. 3, pp. 309-313, 1999.

[181] B. Antebi, G. Pelled, and D. Gazit, "Stem cell therapy for osteoporosis," Curr. Osteoporos. Rep., vol. 12, no. 1, pp. 41-47, 2014, doi: 10.1007/s11914-013-0184-x.

[182] A. J. Engler, S. Sen, H. L. Sweeney, and D. E. Discher, "Matrix elasticity directs stem cell lineage specification.," Cell, vol. 126, no. 4, pp. 677-89, 2006, doi: 10.1016/j.cell.2006.06.044.

[183] F. Guilak, D. M. Cohen, B. T. Estes, J. M. Gimble, W. Liedtke, and C. S. Chen, "Control of Stem Cell Fate by Physical Interactions with the Extracellular Matrix," Cell Stem Cell, vol. 5, no. 1, pp. 17-26, 2009, doi: 10.1016/j.stem.2009.06.016.

[184] W. J. Li et al., "A three-dimensional nanofibrous scaffold for cartilage tissue engineering using human mesenchymal stem cells," Biomaterials, vol. 26, no. 6, pp. 599-609, 2005, doi: 10.1016/j.biomaterials.2004.03.005.

[185] M. J. Dalby et al., "The control of human mesenchymal cell differentiation using nanoscale symmetry and disorder," Nat. Mater., vol. 6, no. 12, pp. 997-1003, 2007, doi: 10.1038/nmat2013.

[186] S. Oh et al., "Stem cell fate dictated solely by altered nanotube dimension," Proc. Natl. Acad. Sci., vol. 106, no. 7, pp. 2130-2135, Feb. 2009, doi: 10.1073/pnas.0813200106.

[187] Y. Chen, Z. Sun, Y. Li, and Y. Hong, "Osteogenic commitment of mesenchymal stem cells in apatite nanorod-aligned ceramics," ACS Appl. Mater. Interfaces, vol. 6, no. 24, pp. 21886-21893, 2014, doi: 10.1021/am5064662.

[188] S. W. Kuo et al., "Regulation of the fate of human mesenchymal stem cells by mechanical and stereo-topographical cues provided by silicon nanowires," Biomaterials, vol. 33, no. 20, pp. 5013-5022, 2012, doi: 10.1016/j.biomaterials.2012.03.080.

[189] H. Y. Chen et al., "Resistive random access memory (RRAM) technology: From material, device, selector, 3D integration to bottom-up fabrication," J. Electroceramics, vol. 39, no. 1-4, pp. 2138, 2017, doi: 10.1007/s10832-017-0095-9.

[190] G. Milano, S. Porro, I. Valov, and C. Ricciardi, "Recent Developments and Perspectives for Memristive Devices Based on Metal Oxide Nanowires," Adv. Electron. Mater., vol. 1800909, 2019, doi: 10.1002/aelm.201800909.

[191] K. Nagashima and T. Yanagida, "16 - Oxide nanowires for nonvolatile memory applications," in Magnetic Nano- and Microwires, Elsevier, 2015, pp. 489-524.

[192] S. S. P. Parkin, M. Hayashi, and L. Thomas, "Magnetic DomainWall Racetrack Memory," Science (80-. )., vol. 320, no. 5873, pp. 190-194, Apr. 2008, doi: 10.1126/science.1145799.

[193] T. Coughlin, R. Hoyt, and J. Handy, "Digital Storage and Memory Technology ( Part 1 )," IEEE, no. November, pp. 1-31, 2017.

[194] J. J. Yang, D. B. Strukov, and D. R. Stewart, "Memristive devices for computing," Nat. Nanotechnol., vol. 8, no. 1, pp. 13-24, 2013, doi: 10.1038/nnano.2012.240

[195] S. I. Kim, J. H. Lee, Y. W. Chang, S. S. Hwang, and K.-H. Yoo, "Reversible resistive switching behaviors in NiO nanowires," Appl. Phys. Lett., vol. 93, no. 3, p. 033503, Jul. 2008, doi: 10.1063/1.2958234.

[196] E. D. Herderick, K. M. Reddy, R. N. Sample, T. I. Draskovic, and N. P. Padture, "Bipolar resistive switching in individual $\mathrm{Au}-\mathrm{NiO}-\mathrm{Au}$ segmented nanowires," Appl. Phys. Lett., vol. 95, no. 20, pp. 20-23, 2009, doi: 10.1063/1.3263733.

[197] Y. C. Huang et al., "Using binary resistors to achieve multilevel resistive switching in multilayer $\mathrm{NiO} / \mathrm{Pt}$ nanowire arrays," $N P G$ Asia Mater., vol. 6, no. 2, pp. e85-7, 2014, doi: 10.1038/am.2013.81.

[198] K. Park and J. S. Lee, "Controlled synthesis of $\mathrm{Ni} / \mathrm{CuOx} / \mathrm{Ni}$ nanowires by electrochemical deposition with self-compliance bipolar resistive switching," Sci. Rep., vol. 6, no. January, pp. 1-6, 2016, doi: 10.1038/srep23069.

[199] J. M. Song and J. S. Lee, "Self-assembled nanostructured resistive switching memory devices fabricated by templated bottom-up growth," Sci. Rep., vol. 6, no. January, pp. 1-7, 2016, doi: $10.1038 /$ srep 18967.

[200] C. Cagli, F. Nardi, B. Harteneck, Z. Tan, Y. Zhang, and D. Ielmini, "Resistive-switching crossbar memory based on $\mathrm{Ni}-\mathrm{NiO}$ core-shell nanowires," Small, vol. 7, no. 20, pp. 2899-2905, 2011, doi: 10.1002/smll.201101157.

[201] A. Al-Haddad et al., "Highly-ordered 3D vertical resistive switching memory arrays with ultralow power consumption and ultrahigh density," ACS Appl. Mater. Interfaces, vol. 8, no. 35, pp. 2334823355, 2016, doi: 10.1021/acsami.6b05424.

[202] Y. P. Ivanov, A. Chuvilin, S. Lopatin, and J. Kosel, "Modulated Magnetic Nanowires for Controlling Domain Wall Motion: Toward 3D Magnetic Memories," ACS Nano, vol. 10, no. 5, pp. 5326-5332, 2016, doi: 10.1021/acsnano.6b01337.

[203] F. Nasirpouri et al., "Geometrically designed domain wall trap in tri-segmented nickel magnetic nanowires for spintronics devices," Sci. Rep., vol. 9, no. 1, p. 9010, 2019, doi: 10.1038/s41598-01945553-w.

[204] J. A. Moreno, H. Mohammed, and J. Kosel, "Effect of Segment length on domain wall pinning in multisegmented $\mathrm{Co} / \mathrm{Ni}$ nanowires for 3D memory applications," J. Magn. Magn. Mater., vol. 484, no. April, pp. 110-113, 2019, doi: 10.1016/j.jmmm.2019.04.002.

[205] M. Schöbitz et al., "Fast Domain Wall Motion Governed by Topology and Ersted Fields in Cylindrical Magnetic Nanowires," Phys. Rev. Lett., vol. 123, no. 21, pp. 1-5, 2019, doi: 10.1103/PhysRevLett.123.217201.

[206] A. Wartelle et al., "Bloch-point-mediated topological transformations of magnetic domain walls in cylindrical nanowires," Phys. Rev. B, vol. 99, no. 2, p. 024433, Jan. 2019, doi: 10.1103/PhysRevB.99.024433.

[207] J. García Fernández, V. Vega Martínez, A. Thomas, V. de la Prida Pidal, and K. Nielsch, "Two-Step Magnetization Reversal FORC Fingerprint of Coupled Bi-Segmented Ni/Co Magnetic Nanowire Arrays," Nanomaterials, vol. 8, no. 7, p. 548, 2018, doi: 10.3390/nano8070548.

[208] E. M. Palmero, F. Béron, C. Bran, R. P. del Real, and M. Vázquez, "Magnetic interactions in compositionally modulated nanowire arrays," Nanotechnology, vol. 27, no. 43, p. 435705, 2016, doi: 10.1088/0957-4484/27/43/435705.

[209] N. Perrissin et al., "A highly thermally stable sub-20 nm magnetic random-access memory based on perpendicular shape anisotropy," Nanoscale, vol. 10, no. 25, pp. 12187-12195, 2018, doi: $10.1039 / \mathrm{c} 8 \mathrm{nr} 01365 \mathrm{a}$

[210] K. Watanabe, B. Jinnai, S. Fukami, H. Sato, and H. Ohno, "Shape anisotropy revisited in single-digit nanometer magnetic tunnel junctions," Nat. Commun., vol. 9, no. 1, p. 663, Dec. 2018, doi: 10.1038/s41467-018-03003-7.

[211] N. Perrissin et al., "Perpendicular shape anisotropy spin transfer torque magnetic random-access memory: Towards sub-10 nm devices," J. Phys. D. Appl. Phys., vol. 52, no. 23, 2019, doi: 10.1088/1361-6463/ab0de4.

[212] J. Spiegel, J. De La Torre, M. Darques, L. Piraux, and I. Huynen, "Permittivity model for ferromagnetic nanowired substrates," IEEE Microw. Wirel. Components Lett., vol. 17, no. 7, pp. 492-494, 2007, doi: 10.1109/LMWC.2007.899303.

[213] A. Encinas, M. Demand, L. Vila, L. Piraux, and I. Huynen, "Tunable remanent state resonance frequency in arrays of magnetic nanowires," Appl. Phys. Lett., vol. 81, no. 11, pp. 2032-2034, 2002, doi: 10.1063/1.1507610.

[214] M. Darques, J. Spiegel, J. De la Torre Medina, I. Huynen, and L. Piraux, "Ferromagnetic nanowire-loaded membranes for microwave electronics," J. Magn. Magn. Mater., vol. 321, no. 14, pp. 20552065, 2009, doi: 10.1016/j.jmmm.2008.03.060.

[215] Y. Lei, L. Li, L. Yuan, and Z. Yue, "Tunable High-Frequency Properties of Co-Ni Ferromagnetic Nanowires Through Composition Modulation," IEEE Trans. Magn., vol. 51, no. 11, pp. 1-6, 2015, doi: 10.1109/TMAG.2015.2440371.

[216] M. Darques, L. Piraux, A. Encinas, P. Bayle-Guillemaud, A. Popa, and U. Ebels, "Electrochemical control and selection of the structural and magnetic properties of cobalt nanowires," Appl. Phys. Lett., vol. 86, no. 7, pp. 1-3, 2005, doi: 10.1063/1.1866636. 
[217] J. De La Torre Medina, J. Spiegel, M. Darques, L. Piraux, and I. Huynen, "Differential phase shift in nonreciprocal microstrip lines on magnetic nanowired substrates," Appl. Phys. Lett., vol. 96, no. 7, pp. 7-10, 2010, doi: 10.1063/1.3313942.

[218] C. E. Carreón-González, J. De La Torre Medina, L. Piraux, and A. Encinas, "Electrodeposition growth of nanowire arrays with height gradient profiles for microwave device applications," Nano Lett., vol. 11, no. 5, pp. 2023-2027, 2011, doi: 10.1021/n12003939.

[219] V. Van Kerckhoven, L. Piraux, and I. Huynen, "A laser-assisted process to produce patterned growth of vertically aligned nanowire arrays for monolithic microwave integrated devices,"

Nanotechnology, vol. 27, no. 23, 2016, doi: 10.1088/09574484/27/23/235301

[220] Y. Lei, Z. Chen, and L. Li, "Microwave properties of ferromagnetic nanowire arrays patterned with periodic and quasi-periodic structures," J. Appl. Phys., vol. 117, no. 17, pp. 1-5, 2015, doi: 10.1063/1.4917187.

[221] R. Ramirez-Villegas, I. Huynen, L. Piraux, A. Encinas, and J. De La Torre Medina, "Configurable Microwave Filter for Signal Processing Based on Arrays of Bistable Magnetic Nanowires," IEEE Trans. Microw. Theory Tech., vol. 65, no. 1, pp. 72-77, 2017, doi: 10.1109/TMTT.2016.2614926. 